Document downloaded from:

http://hdl.handle.net/10251/153689

This paper must be cited as:

Diego-Mas, JA.; Garzon Leal, D.; Poveda Bautista, R.; Alcaide Marzal, J. (2019). Userinterfaces layout optimization using eye-tracking, mouse movements and genetic algorithms. Applied Ergonomics. 78:197-209. https://doi.org/10.1016/j.apergo.2019.03.004

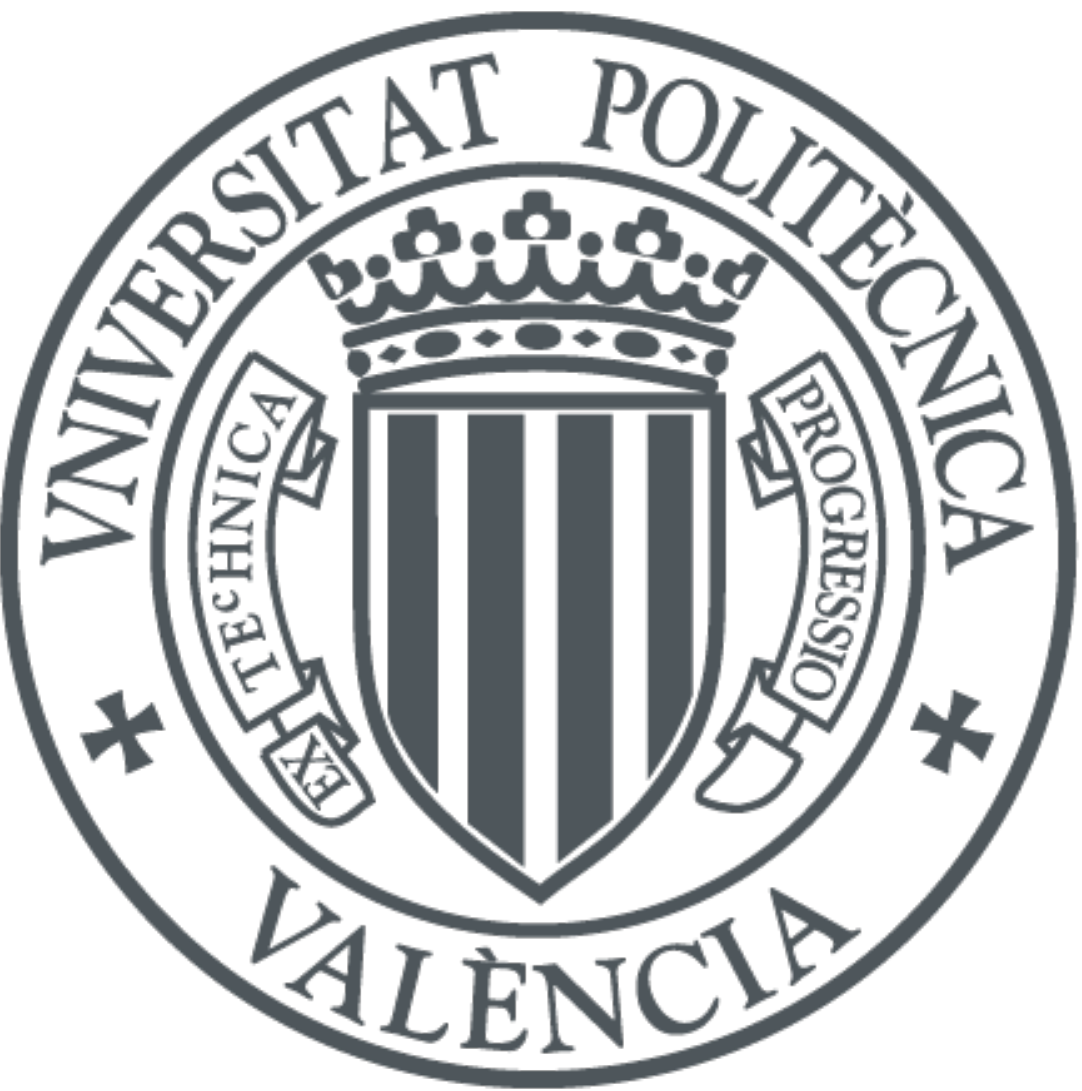

The final publication is available at

https://doi.org/10.1016/j.apergo.2019.03.004

Copyright Elsevier

Additional Information 


\title{
User-interfaces layout optimization using eye-tracking, mouse movements and genetic algorithms (author version).
}

Jose Antonio Diego-Mas ${ }^{\mathrm{a}^{*}}$, Diana Garzon-Leal ${ }^{\mathrm{b}}$, Rocio Poveda-Bautista ${ }^{\mathrm{c}}$ and Jorge AlcaideMarzal $^{\mathrm{a}}$

${ }^{\text {a }}$ I3B, Institute for Research and Innovation in Bioengineering, Universitat Politecnica de Valencia, Camino de Vera s/n, 46022 Valencia, Spain. jodiemas@dpi.upv.es

b Universidad del Bosque, Av. Cra 9 No. 131 A - 02, Bogotá, Colombia. dgarzonl@unbosque.edu.co

c INGENIO (CSIC-UPV), Universitat Politecnica de Valencia, Camino de Vera s/n, Valencia 46070, Spain ropobau@upvnet.upv.es

*Corresponding author. Tel.: +34 963877 000. E-mail address: jodiemas@dpi.upv.es (J.A. Diego-Mas)

\begin{abstract}
Establishing the best layout configuration for software-generated interfaces and control panels is a complex problem when they include many controls and indicators. Several methods have been developed for arranging the interface elements; however, the results are usually conceptual designs that must be manually adjusted to obtain layouts valid for real situations. Based on these considerations, in this work we propose a new automatized procedure to obtain optimal layouts for software-based interfaces. Eye-tracking and mouse-tracking data collected during the use of the interface is used to obtain the best configuration for its elements. The solutions are generated using a slicing-trees based genetic algorithm. This algorithm is able to obtain really applicable configurations that respect the geometrical restrictions of elements in the interface. Results show that this procedure increases effectiveness, efficiency and satisfaction of the users when they interact with the obtained interfaces.
\end{abstract}

Keywords: interfaces; layout; eye-tracking; genetic algorithms 


\section{Introduction}

Human-machine interfaces can be as simple as single displays or controls, or as technologically advanced as a multi-touch-enabled control panel (CP). Over the years, machines and processes controlled through CPs have become more and more complex. Significant progresses have been made in process monitoring and control instrumentation; consequently, the number of indicators and controls in CPs is constantly increasing. Nowadays, classical interfaces and CPs have been replaced by visual display units (VDUs) in control rooms of industrial processes. For example, in power plants or mines (Carvalho et al., 2008; Horberry et al., 2010; Hwang et al., 2009; Lin and Wu, 2010), or in public transports such as trains, ships or planes (Degani et al., 1992). In the same way, VDUs are present in processes that need a lot of data to be simultaneously presented, like stock market trading, health care, traffic, etc. (Raeisi et al., 2016). Progressively, dedicated "hard” controls and displays have been replaced by reconfigurable “soft” elements on VDUs (Degani et al., 1992).

The design of the CPs, and the different types of controls and displays that make them up, have been widely studied in past years using principles of human information processing (Alluisi and Morgan, 1976) looking for efficiency, safety, and ergonomics (Kantowitz and Sorkin, 1983; Kroemer et al., 2001; Sanders and McCormick, 1993). Well-known rules have been established for selecting and arranging different sorts of controls and displays in interfaces according to diverse needs and conditions (International Organization for Standardization, 2008; Kroemer et al., 2001; Stewart, 1995). For example, ISO 11064-5 contains a checklist to verify the implementation of design principles as well as a process description for display and control specification.

Unlike the old analogic CPs, software-generated interfaces offer more flexibility and adaptability. The configuration and the layout of the CP can be adapted to operators' requirements and to the task being developed. For example, in a software-based CP, controls and displays are usually presented in windows that can be resized or rearranged. Therefore, employing software-generated interfaces can achieve a high level of efficiency, satisfying personal requirements at the same time (Nachreiner et al., 2006).

As the interfaces become more complex and flexible, new opportunities and problems appear. Due to the adaptability of the software-generated interfaces, it is possible to adapt the layout of the CPs to the requirements of the task developed (Gajos et al., 2006a), or to the user of the CP at each moment (Todi et al., 2018). However, establishing the best configuration for a CP including many controls and indicators is a complex problem. The convenient position and size of each element must be established to optimize the task performance based on several criteria, while multiple restrictions must be considered to maintain the functionality of the CP. Fortunately, new computational interaction methods help to solve these problems. A review on these new techniques can be found in Oulasvirta et al. (2018).

Several methods have been developed for arranging interface elements in CPs (Chapanis, 1996; Oulasvirta, 2017a). Process-analysis techniques perform a functional analysis of the interaction studying different steps of the process. Based on this analysis, flow diagrams or flow charts of the interaction are created and can be used to establish the CP layout (Alppay and Bayazit, 2015). Link analysis (Lin and Wu, 2010; Wickens et al., 2003) is a process-analysis technique widely used to improve user interfaces. This technique analyses the relationships between the elements of CPs and represents the intensity of these relationships using statistical values in the links between the corresponding elements. 
Usually, the results of the application of these techniques are schematic charts, interaction tables or sequence diagrams that can be used to optimizing the arrangement of the elements in the interface. Initially, CPs layouts were created manually by experts relying on experience and design principles; however, when the complexity of the problem increased, computer programs and computational models were developed to assist designers (Foley et al., 1991; Kim and Foley, 1990; Oulasvirta et al., 2018; Wu and Liu, 2009). The most common approach is to build a quantitative model of the human-interface interaction, and subsequently, to look for the configuration that provides the best model performance (Francis, 2000). Due to the complexity of the problem, techniques like linear programming (Holman et al., 2003), hill-climbing (Francis, 2000), simulated annealing (Francis, 2000; Geman and Geman, 1984) and different heuristics and metaheuristics have been used.

These procedures present two main problems. Firstly, the results are conceptual designs that require manual adjustments to obtain a layout valid for a real situation. Although these general arrangements indicate the optimal position for each component, there are restrictions such as the size or the shape of the controls and displays, or the total available space in the $\mathrm{CP}$, which the solution obtained from these procedures do not consider. Therefore, it is necessary to introduce changes in the initial optimal arrangement, and the solution finally implemented could be far from optimal. On the other hand, some of the methods used to gather data can affect the behavior of the observed user, can present some subjectivity in the measurements or can be imprecise (Alppay and Bayazit, 2015). These problems make it difficult to obtain significant results or to generalize the findings.

Avoiding these drawbacks implies to use a resolution technique that considers the geometrical restrictions of the CPs during the search for the optimal layout. In this way, the obtained solution can be directly implanted without the need for manual adjustments that distort the layout. On the other hand, it is necessary to use a simpler, less time-consuming and less invasive method to gather data during the observation of the users' activities.

Achieving these objectives can simplify the process of obtaining new optimal layouts, and take advantage of the adaptability of software-based CPs.

Based on these considerations, in this work we propose a new automatized procedure to obtain optimal layouts for software-based CPs. Data from input devices (e.g. the path of the cursor over the screen and the clicks of the mouse's buttons) are collected while the users perform usual tasks by means of the CP. Simultaneously, eyetracking devices are used to collect data on the users' gaze over the CP. On the other hand, a quantitative model of the human-interface interaction is built using common principles in the arrangement of elements in CPs (Sanders and McCormick, 1993). Based on these principles and using the data collected, links between the interface elements are established and represented in a link table (Wickens et al., 2003). Finally, a slicing-trees based genetic algorithm (Holland, 1975; Srinivas and Patnaik, 1994) is used for optimizing the layout of the controls and displays in CPs. This metaheuristic, based on the slicing tree structure (Tam, 1992), is specially efficient in the search for geometrically acceptable solutions (Diego-Mas et al., 2017, 2008), avoiding the need for manual adjustments of the final layout.

Eye-tracking is a particularly relevant technique for assessing user-machines interactions and mental workload during the execution of the tasks, that has been previously used for analyzing interactions between operators and CPs (Karn et al., 1999; Martin et al., 2011; Stainer et al., 2013; Starke et al., 2015; Wedel, 2017). Mainly, these previous works deal with mental workload evaluation during task execution. Some of them use eye-tracking to 
compare the operators' workflows or to evaluate the correspondence between eye movement and the goal structure of a task. Therefore, eye-gaze is used to evaluate the working conditions or the adaptation level of a CP to the task developed. However, the obtained data are not used to improve the design of the CPs. One of the main contribution of our work lies in the integration of the eye-tracking data into the design of the optimal layout of the CPs.

The following sections describe the developed procedure and its application to a case study. Section 2 describes the design principles and data gathered to generate the layouts of the CPs, as well as the way used to measure the performance of the users-CP interaction. Section 3 shows the problem definition and the GA designed to find the layout that best match the established criteria respecting the geometrical restrictions. A case study is presented in Sections 4 and its results are shown in Section 5. Finally, the obtained results are discussed in Section 7.

\section{Design principles, data collection and performance measuring}

The basic design principles for the arrangement of interface elements established in Sanders \& McCormick (1993) have been widely accepted and used for the design of interfaces. The principle of sequence of use specifies that the elements of the interface used in operational sequences must be located with regards to these sequences. In the same way, the proximity compatibility principle (Wickens and Carswell, 1995) demands that elements relevant to a common task or mental operation should be rendered close together. The frequency of use of each element present in a CP must also be considered. Therefore, controls and indicators frequently accessed must occupy convenient locations. The position and area occupied by these elements should be defined to facilitate accessibility and usability.

These design principles must be transformed in design requirements. Usually, the following design requirements are established:

- the elements of the $\mathrm{CP}$ that are frequently accessed sequentially must be close to each other in the arrangement;

- the elements of the $\mathrm{CP}$ that are accessed together in a common task must be close to each other;

- the elements shall be sized according to their frequency of access and use;

The implementation of these design requirements makes it necessary to define an access to an element of the CP. A software-based CP operator can access an element in two ways; obtaining information from an indicator or a display, or using a control (for example clicking the mouse on it). It is a common task for CPs operators to use a control while looking a display in order to read the response of the controlled process. This example is a case in which two elements are sequentially accessed; the control is accessed using the pointing device and the display using the gaze. For this reason, both actions, using the pointing device and looking at an element, will be considered an access in this work. In this way, to look consecutively at two elements, or using a control while looking another element, will both be considered cases of sequential accesses between two elements.

During the CP operation, the number and sequence of accesses to its elements must be captured. In order to detect actions on the controls, the software events of the pointing device can be registered. Concurrently, an eyetracking device collects data on the operators' gaze. The obtained data be used to optimize the arrangement of the elements of the $\mathrm{CP}$ following the design requirements previously mentioned. This optimization process requires the definition of a quantitative fitness function that measures the degree in which a proposed layout 
accomplishes the design requirements. The objective of the optimization process is to find the layout that maximizes this fitness function.

The quality of the arrangements of the elements in a $\mathrm{CP}$ depends on the human-CP interaction performance. Once the layout that best fits the design requirements has been obtained, its performance must be compared with other arrangements to validate the capability of the procedure presented in this work to find optimal layouts. The human-interface interaction performance should rather consider efficacy, efficiency and satisfaction in the development of the tasks (International Organization for Standardization, 1998), and different indicators must be used to measure each of these aspects (Frøkjær et al., 2000). In the case study of this work, we will measure the performance of a proposed layout while a group of operators performs the same series of tasks. In this way, it will be possible to compare the number of errors committed, the time consumed to carry them out, and the users' satisfaction measured by attitude rating scales.

\section{Material and methods}

The procedure proposed in this work has two stages. In the first stage, operators are monitored while using the CP to develop several predefined tasks in order to gather mouse and eye-tracking data. Using these data, and based on the design requirements exposed in the previous section, links between the CP elements are established and represented in a link table (Wickens et al., 2003). The value of the link between two components represents the need for closeness between them. In the second stage, a GA is used to obtain the optimal layout considering the link table and the geometrical restrictions imposed to the elements of the layout.

\subsection{Problem definition}

Given a CP to be optimized like that shown in Figure 1a, let $\mathbf{R}$ be its geometric ratio (height divided by width). Generally, $\mathbf{R}$ matches the geometric ratio of the screen that will show the CP. The number of elements to be distributed in the CP will be called $\mathbf{n}$, and $\mathbf{a}_{\mathbf{i}}$ will be the percentage of the area of the CP occupied by each element $\mathbf{i}$. These areas are considered to be rectangular and with flexible geometry. Flexible geometry means that the geometric ratio of the area allocated for the element may vary within certain limits (geometric constraints). These limits ( $\mathbf{r}_{\text {min_i }}$ and $\mathbf{r}_{\text {max }} \mathbf{i}$ ) depends on the kind of control or indicator that occupies the area, and they must be established considering the functionality of the element. For example, let $\mathbf{a}$ be the area occupied by a slider bar in a CP, and $\mathbf{r}_{\min }=0.16$ and $\mathbf{r}_{\max }=6.00$ the minimum and maximum geometric ratios permissible for this element. Figure $1 \mathrm{~b}$ shows the different permissible shapes for the slider bar. While the area a remains constant, the ratio between the height and width of the slider varies within $\mathbf{r}_{\min }$ and $\mathbf{r}_{\text {max }}$. The area and the geometric limits of the elements must be assigned considering that certain controls or indicators require a specific orientation. For example, elements with both ratio limits lower than one have horizontal orientation (Figure 1b), while ratios higher than one force vertical orientations.

Rearranging the elements of the CP can imply variations in the size of the elements to avoid overlapping elements or empty spaces in the screen. However, this variation of the initial area must be limited to avoid that some elements become unrealistic small, or that very frequently accessed elements occupy most of the CP. Hence, minimum and maximum areas of the elements ( $\mathbf{a}_{\text {min_i }}$ and $\left.\mathbf{a}_{\text {max }} \mathbf{i}\right)$ are defined. Hence, the area $\left(\mathbf{a}_{\mathbf{i}}\right)$ of one element can be varied in [amin_i $\mathbf{a}$ amax_i $]$. 
Summarizing the definition of the problem, $\mathbf{n}$ elements must be rearranged in a CP with a geometric ratio $\mathbf{R}$. The initial area of each element $\mathbf{i}$ is $\mathbf{a}_{\mathbf{i}}$, its geometric ratio can be varied between $\mathbf{r}_{\mathbf{m i n}} \mathbf{i}$ and $\mathbf{r}_{\mathbf{m a x} \_} \mathbf{i}$, and its area must be in $\mathbf{a} \mathbf{i} \pm \mathbf{a} \mathbf{a} i \mathbf{i}_{-} \mathbf{i}$.

a)

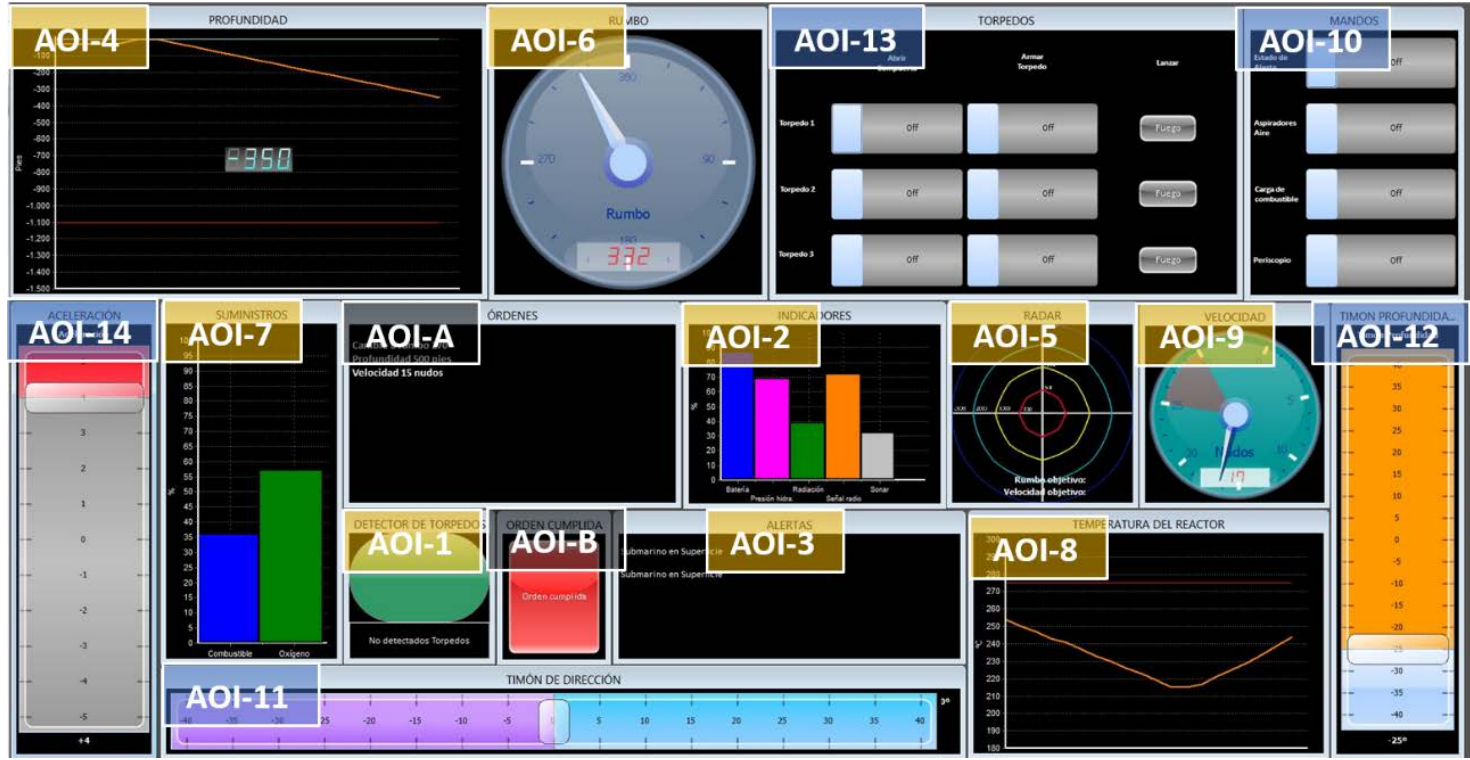

b)

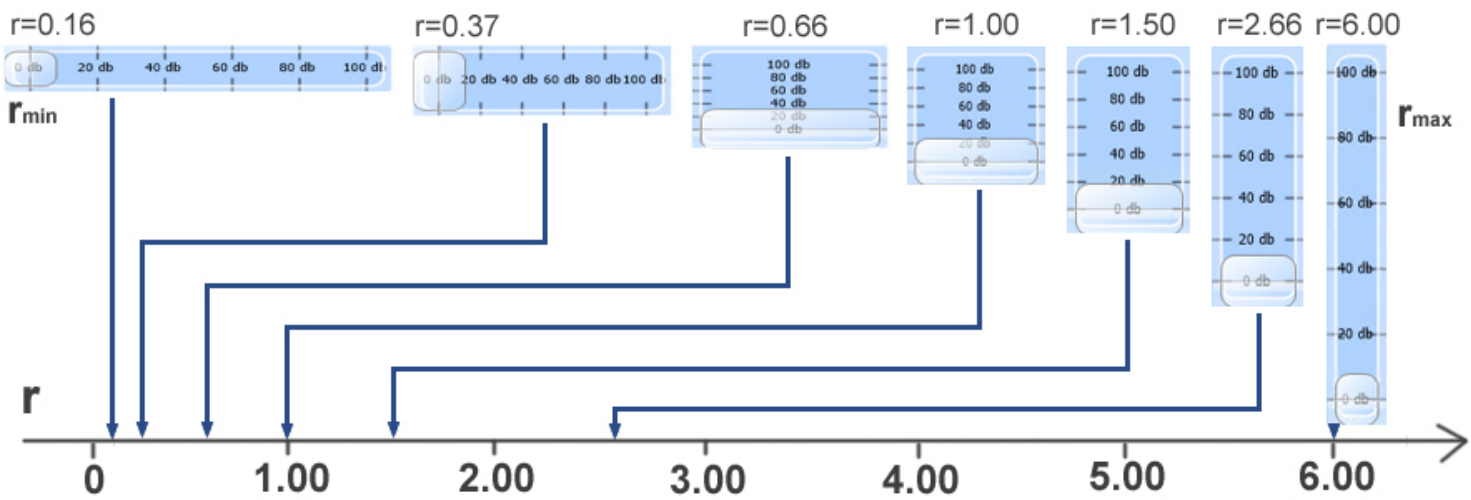

Figure 1: (a) Areas of interest (AOI) defined for each element of the interface. AOI-1 to AOI-9 were shaped over indicators, and AOI-10 to AOI-14 over controls. AOI-A and AOI-B were defined over elements related to the communications systems. (b) Variation of the shape of an element of a CP between its min and max geometric ratios. Ratios lower than one produce horizontal orientations. Ratios greater than one result in vertical orientations.

\subsection{Data collection}

In the first stage, a software running on a PC is used to simulate the target CP on a VDU equipped with an unobtrusive screen-based eye-tracking device. The position, size and functionality of the elements are the same as those of the target CP's elements. The software detects the actions over the controls and a software model of the controlled process calculates and shows the responses in the corresponding indicators of the CP.

For the analysis of eye movements, each element of the CP is defined as an area of interest (AOI) (Holmqvist et al., 2011). AOIs are used in eye-tracking studies to link eye-movement measures to parts of the stimulus used (Hessels et al., 2016). AOI’s are defined drawing boxes around elements on the screen (Figure 1a) and naming 
them using the eye-tracker software, and are meant to help with doing quantitative analysis, calculating how often and how long these elements were looked at (Pernice et al., 2009).

Several operators with experience in operating the target CP are selected and asked to complete the same sequence of tasks on the CP. The task to be performed by the operators depends on the goal of the study and the characteristics of the controlled process. The sequence of tasks may include, for example, the most common tasks of the controlled process, critical tasks in which no mistakes should be made or tasks that should be done in a short time. While the operator performs the job, the software registers the time devoted to each task, the number of mistakes made, the mouse path on the screen and the mouse-click events (including the number of clicks and the position of the cursor during each click). Simultaneously, the eye-tracker registers the position of the gaze of the operator.

When all the operators have performed the sequence of tasks, eye-tracking data of each operator are analyzed to obtain the percentage dwell time (PDT) (Blascheck et al., 2014) for each AOI. PDT is the percentage of time that the operators eyes remain in one AOI relative to the other AOIs (Helleberg and Wickens, 2003; Wickens C. D., 2008; Wickens et al., 2002). Besides, mouse-click events are examined registering how many times each AOI has been clicked, and calculating the percentage of clicks over each AOI relative to the total number of clicks. These data are used to obtain the transitions of gaze between the AOIs of the CP. A transition is a movement of the gaze from one AOI to another (Goldberg and Kotval, 1999). A common visualization technique of transitions between AOIs is the transition matrix (Blascheck et al., 2014; Goldberg and Kotval, 1999). Transition matrix arranges AOIs horizontally in rows and vertically in columns. Each element of the matrix $T_{i j}$ has the value of the number of times the transitions from $\mathrm{AOI}_{\mathrm{i}}$ to $\mathrm{AOI}_{\mathrm{j}}$ occurred (one-way transition matrix), or the number of times the transitions between $\mathrm{AOI}_{\mathrm{i}}$ and $\mathrm{AOI}_{\mathrm{j}}$ occurred regardless of direction of the transition (two-way transition matrix) (Harris et al., 1986).

As has been mentioned in Section 2, we have considered two kinds of sequential accesses between the elements of a CP. The first kind of sequential access is the gaze transition between two elements (gaze-gaze sequential accesses). The operator looks at one element and then at another one. The second class occurs when the operator of the CP interacts with an element using the mouse, while looking at another element simultaneously (gazemouse sequential accesses). To capture this kind of sequential accesses, mouse-click events are examined registering the cases in which the click is made on an AOI while the operator is looking at another one. The data from all the operators are used to create a two-way transition matrix, in which each element has the value $\mathrm{X}_{\mathrm{ij}}=$ $\mathrm{T}_{\mathrm{ij}}+\mathrm{C}_{\mathrm{ij}}$. In this expression, $\mathrm{T}_{\mathrm{ij}}$ is the average number of times the transitions between $\mathrm{AOI}_{\mathrm{i}}$ and $\mathrm{AOI}_{\mathrm{j}}$ occurred, and $\mathrm{C}_{\mathrm{ij}}$ is the average number of clicks over one of these AOIs (i or $\mathrm{j}$ ) while the operator is looking at the other. The diagonal of the matrix is always empty because transitions within AOIs cannot occur. Since the matrix is symmetric, only the values under diagonal are represented. This transition matrix will be used as the link table that expresses the need for closeness between the elements of the CP.

\subsection{A genetic algorithm to optimize the $\mathrm{CP}$ layout}

To obtain the best arrangement of a group of elements (controls and indicators) in a VDU of known proportions is a case of a quadratic assignment problem (Karrenbauer and Oulasvirta, 2014), or facility layout problem (FLP) (Kusiak and Heragu, 1987). The FLP is a combinational optimization problem that studies the distribution of a 
set of components with known and unequal area in a flat surface of given dimensions. The elements to be arranged interact with each other; therefore, the layout of the elements on the flat area must be obtained in such a way that costs related to these interactions are keep to a minimum.

There are not exact procedures to solve FLPs if the number of elements to be distributed is high. Therefore metaheuristic techniques are used to obtain solutions to the problem (Zhu et al., 2017). Metaheuristics commonly used to solve FLPs are memetic algorithms (Merz and Freisleben, 1999), particle swarm optimization (HosseiniNasab and Emami, 2013; Önüt et al., 2008), tabu search (James et al., 2009; McKendall and Liu, 2012), simulated annealing (Kulturel-Konak and Konak, 2015; Saifullah Hussin and Stützle, 2014) or genetic algorithms (GAs) (Diego-Mas et al., 2009; El-Baz, 2004; Küçükoğlu and Öztürk, 2017).

GAs are widely used in optimizing user interfaces for human performance (Oulasvirta, 2017a, 2017b). Within this class of algorithms, slicing-trees based genetic algorithms (Holland, 1975; Srinivas and Patnaik, 1994) are specially efficient to obtain geometrically acceptable solutions to FLPs with strict geometric constrains (DiegoMas et al., 2008), avoiding the need for manual adjustments of the final layout. GAs explore the solution space of the problem performing a stochastic guided search based on the evolution of a set (population) of structures (chromosomes) (Goldberg, 1989). Each chromosome represents a solution to the problem. The population of chromosomes is evaluated using a fitness function to measure its suitability for the requirements of the problem. Based on the fitness of each chromosome, a new population that inherit the best characteristics of their predecessors is obtained. The new population is the result of several transformations guided by some genetic operators (generally selection, crossover and mutation), which combine or alter the chromosomes obtaining new solutions. This iterative procedure is repeated a predefined number of iterations or until another stop criterion is reached.

The CP layouts obtained using these methods must be applicable in real practice. Therefore, the geometrical restrictions of elements that form the CP must be respected. Solutions that do not conform to these limits must be considered unacceptable. For this reason, we propose to use a slicing-trees based genetic algorithm to obtain the CP layouts. This GA uses slicing trees to generate the solutions. In our case, a slicing tree is a rooted binary tree in which each leaf is assigned a unique integer corresponding to the identifier of an element to be arranged in the CP. Each internal node represents the way (vertical or horizontal) a rectangular partition of the VDU is cut, allocating the elements in each branch of the node on each side of the partition. Figure 2 shows two slicing trees used to arrange five elements in a VDU recursively cutting the VDU space. Each leaf of the trees corresponds to one element of the CP. Each internal node represents the way a rectangular partition of the VDU is cut (V vertical, $\mathrm{H}$ horizontal), allocating the elements in each branch of the node in each side of the partition. Partitions are generated descending recursively throughout the tree. If the partition is horizontally cut, the elements in the left sub-tree and right sub-tree are allocated in the upper and lower side of the partition respectively. If the partition is vertically cut, the elements of the $\mathrm{CP}$ in the left sub-tree and right sub-tree are allocated in the left and right side of the cut respectively. The calculation of the cut point is straightforward since the areas of the elements to be allocated in each partition are known. Different layouts can be generated by modifying the type of cut that is made in the internal nodes or the structure of the tree.

The proposed GA operate in two phases. In the first one, the algorithm looks for an optimum slicing tree. The trees are generated by applying clustering techniques (using the average linkage method) considering the 
requirements of the different elements to be near each other, and the geometric potential of the slicing trees (Diego-Mas et al., 2008). The geometric potential is an indicator of the capacity of a slicing tree to generate geometrically acceptable solutions. In the second phase, using the previously obtained slicing tree, the algorithm looks for the optimum layout by changing the kind of cuts made in the nodes of the optimal tree. A detailed description of the operation of this kind of algorithms can be found in Diego-Mas et al. (2008).
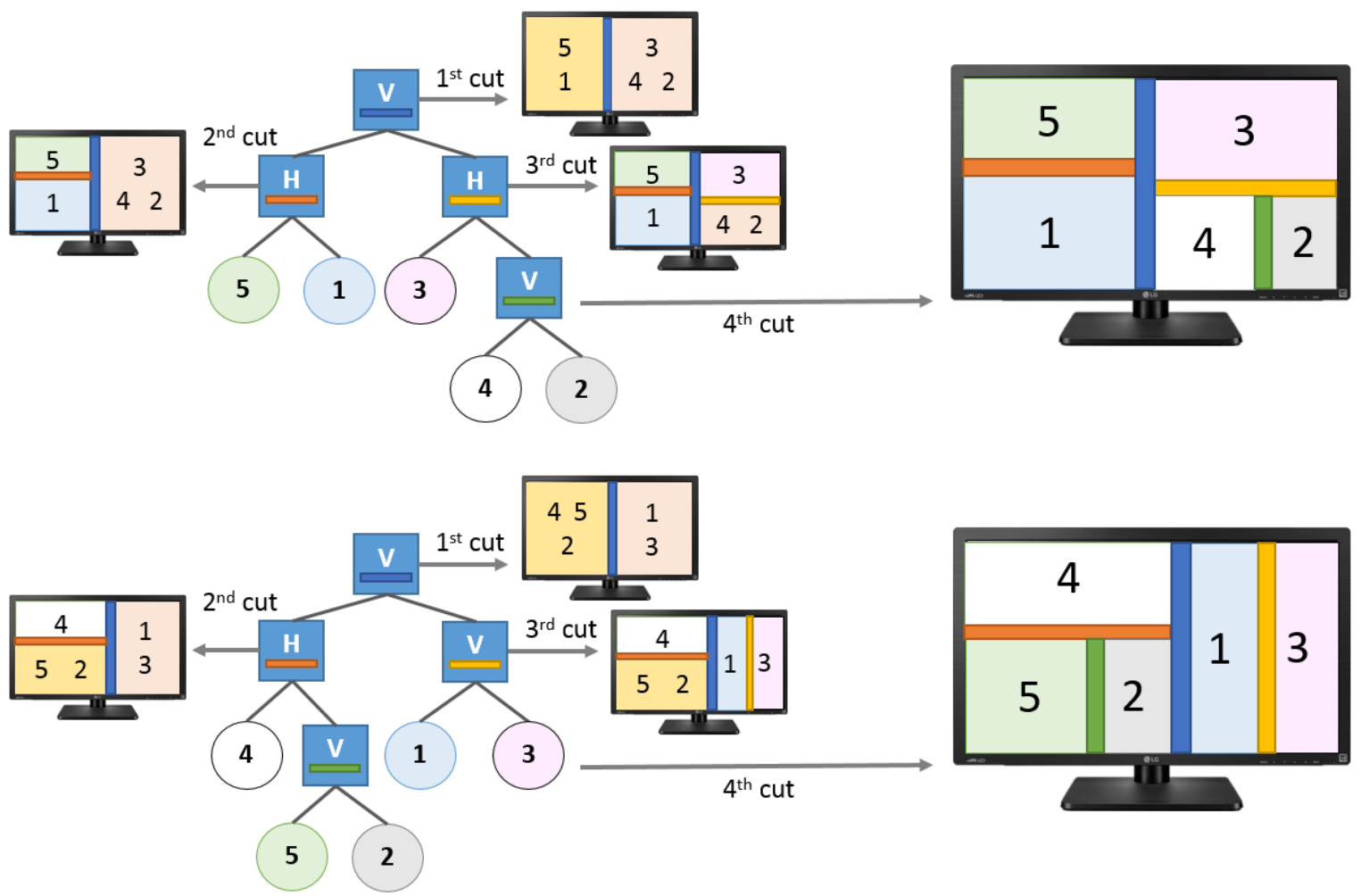

Figure 2: Generating CP layouts in a VDU from slicing trees. Each leaf of the trees corresponds to one element of the CP. Each internal node represents the way a rectangular partition of the VDU is cut (V vertical, $\mathrm{H}$ horizontal), allocating the elements in each branch of the node in each side of the partition. Modifications of the type of cut made in the internal nodes or the structure of the tree will generate different layouts.

\subsubsection{Fitness function}

In a FLP, the elements must be arranged in such a way that costs related to the interactions between them are keep to a minimum, and the geometrical restrictions are respected. This cost is the fitness function that the GA must minimize. We define the cost (C) of a layout for the elements of a CP as shown in Equation 1. In this equation, $\mathbf{n}$ is the number of elements to be distributed in the CP. The total cost is composed of two terms: the relational cost and the geometrical cost. The relational cost (first term in the second member of the equation) is the cost due to the intensity of the relationship between each pair of elements and the distance between them in the layout. The geometrical cost (second term in the second member) measures the degree in which the geometrical restrictions of the elements are respected in the evaluated layout.

$$
C=R c \sum_{i=1}^{n} \sum_{j=1}^{n} d_{i j} x_{i j}+G c \sum_{i=1}^{n} g_{i} \quad \text { Equation } 1
$$


To optimize a CP layout, the elements that are frequently accessed sequentially must be close to each other in the arrangement. The intensity of the relationship between two elements of a CP is measured as the number of transitions between these elements. Therefore, each $\mathbf{x}_{\mathbf{i j}}$ in the equation 1 is the corresponding value in the transition matrix. Consequently, the relational cost is calculated as the product of the number of transitions between each pair of elements $\left(\mathbf{x}_{\mathbf{i j}}\right)$ and the distance between them in the layout $\left(\mathbf{d}_{\mathbf{i j}}\right)$. The distance between elements is usually measured as the euclidean distance between the geometric centers of the elements (Tompkins et al., 2010).

In the geometrical cost, $\mathbf{g}_{\mathbf{i}}$ measures the compliance with the geometric constraints of the element $\mathbf{i}$ in the layout of the CP. As pointed out in Section 3.1, the area occupied by an element in the CP is considered to be rectangular and with flexible geometry (the geometric ratio of the element $\mathbf{i}$ may vary between $\mathbf{r}_{\mathbf{m i n} \_} \mathbf{i}$ and $\mathbf{r}_{\mathbf{m a x}} \mathbf{i}$ ). To measure the compliance with this condition, $\mathbf{g}_{\mathbf{i}}$ value is 0 if the geometric ratio of the element $\mathbf{i}$ in the layout is within the range [ $\left.\mathbf{r}_{\min \_} \mathbf{i}, \mathbf{r} \mathbf{m a x}_{\mathbf{i}}\right]$. Otherwise, $\mathbf{g}_{\mathbf{i}}$ value increases proportionally to the distance to the nearest end of that range.

Relational and geometrical costs are weighted using the Rc and Gc coefficients. By varying these coefficients, the designer is able to give more or less importance to each criterion. Finally, more terms can be added to Equation 1 to consider other criteria. For example, to allow that some elements, like panic buttons or emergency alarms, occupy special position in the layout of the CP.

\section{Experimental application}

An experiment was performed to verify the operation of the proposed procedure. A CP for controlling common processes in a ship was implemented using a simulation software (Figure 1a). The software detects the actions over the controls and a model of the controlled process calculates and shows the responses in the corresponding indicators of the CP. The CP was composed of 16 resizable child windows inside a parent window that occupied the whole screen of the VDU. Each child window contained one control to act on the process (i.e. speed controls, engines controls, communications controls...) or one indicator showing information about the process (i.e. speed, direction, route, radar, engines state, communications...). Four types of indicators were used: numeric displays, dials, graphs and gauges. The controls were slider bars and buttons.

Eye-tracking devices have been improved dramatically in recent years. Nowadays, apart from using expensive specialized devices, low-cost eye-tracking software can work on commodity hardware such as mobile phones, tablets or web-cams without the need for additional sensors or devices (Cai et al., 2017). Selecting the appropriate device for each application must considers some advantages and disadvantages of both kind of devices, like accuracy, robustness, need of calibration process or price (Titz et al., 2018). In this work, a Tobii TX300 Eye-Tracker was employed for the experiment. This eye-tracker collects data look at $300 \mathrm{~Hz}$ with an accuracy rate of $0.5^{\circ}$ and $<0.03^{\circ} \mathrm{drift}$, and is integrated in a 23', TFT monitor that was used as VDU for showing the CP. Tobii Pro Studio analysis software that was used to record and analyze the eye-tracking data and perform the calibration process.

Using this software, an AOI was defined for each child window of the CP. AOI-1 to AOI-9 corresponded to indicators, and AOI-10 to AOI-14 were defined over controls. Two AOIs named AOI-A and AOI-B were defined over elements related to the communications systems. The initial layout of the CP and the AOIs 
distribution are shown in Figure 1a. The ratio and the area of each element, as a percentage of the total VDU, are shown in the second and the sixth columns of Table 1. Minimum and maximum values for the areas and ratios of each element were established. These values depend on the kind and functionality of the element. For example, minimum and maximum ratios of the vertical sliders must be greater than one, while the ratios of the horizontal sliders must be lower than one. In the same way, some indicators need small areas for show information while some controls need bigger areas to facilitate their operation. Minimum and maximum values for areas and ratios are shown in Table 1.

\begin{tabular}{|c|c|c|c|c|c|c|c|c|}
\hline \multirow[b]{2}{*}{ ID } & \multicolumn{4}{|c|}{ Area (\%) } & \multicolumn{4}{|c|}{ Ratio } \\
\hline & Initial & Min & Max & Final & Initial & Min & Max & Final \\
\hline AOI-1 & 2.21 & 2 & 6 & 2.30 & 1.02 & 0.20 & 2.00 & 0.25 \\
\hline AOI-2 & 5.03 & 2 & 6 & 2.38 & 0.79 & 0.20 & 2.00 & 0.26 \\
\hline AOI-3 & 4.95 & 2 & 6 & 3.59 & 0.44 & 0.20 & 1.00 & 0.39 \\
\hline AOI-4 & 12.81 & 2 & 14 & 7.08 & 0.62 & 0.20 & 5.00 & 4.49 \\
\hline AOI-5 & 3.64 & 2 & 6 & 3.62 & 1.10 & 0.90 & 1.10 & 0.97 \\
\hline AOI-6 & 7.57 & 3 & 8 & 4.82 & 1.07 & 0.70 & 1.43 & 0.73 \\
\hline AOI-7 & 5.95 & 2 & 6 & 2.34 & 2.04 & 1.00 & 5.00 & 3.76 \\
\hline AOI-8 & 8.30 & 2 & 14 & 2.42 & 0.69 & 0.20 & 1.00 & 0.27 \\
\hline AOI-9 & 3.63 & 3 & 8 & 7.08 & 1.09 & 0.20 & 5.00 & 4.49 \\
\hline AOI-10 & 5.81 & 4 & 14 & 11.86 & 1.35 & 1.00 & 2.00 & 1.30 \\
\hline AOI-11 & 6.83 & 4 & 14 & 10.82 & 0.12 & 0.10 & 0.50 & 0.32 \\
\hline AOI-12 & 6.00 & 4 & 14 & 10.69 & 3.41 & 2.00 & 10.00 & 2.97 \\
\hline AOI-13 & 12.81 & 4 & 14 & 9.59 & 0.61 & 0.50 & 2.00 & 0.93 \\
\hline AOI-14 & 6.00 & 4 & 14 & 10.69 & 3.15 & 2.00 & 10.00 & 2.97 \\
\hline AOI-A & 6.46 & 2 & 8 & 4.75 & 0.61 & 0.20 & 2.00 & 0.30 \\
\hline AOI-B & 2.00 & 2 & 8 & 5.98 & 1.35 & 0.20 & 2.00 & 0.37 \\
\hline
\end{tabular}

Table 1. Characteristics of the elements to be distributed in the case study. Columns Initial and Final show the area and the geometric ratio of each AOI in the initial and final layouts. Columns Mix and Max show the geometric constraints of each AOI.

A sequence of 20 tasks to be performed on the CP was established including common and critical operations, and 20 users with experience in the use of this kind of CPs were selected to perform the experiment.

\subsection{First experimental session}

All 20 users participated in the first experimental session. The objective of the experiment and the basic procedures to use the CP were explained to participants. They performs some basic operations using the $\mathrm{CP}$ during 15 minutes to be familiarized with its functioning. After that, participants were asked to perform the previously established sequence of tasks using the CP arranged as in Figure 1a. The goal of each task was shown to the participant in a display occupying the AOI-A. When the participant considered that the task was completed he used the control in AOI-B to warn the system and the next task was shown in the AOI-A display. Finally, the participants completed the After-Scenario Questionnaire (ASQ) (Lewis, 1995) to measure the users' satisfaction. This questionnaire consists of three questions that the respondent must answer using a 7 point Likert scale, ranging from 1 (strongly agree) to 7 (strongly disagree). The three questions are: 1- Overall, I am satisfied 
with the ease of completing this task, 2- Overall, I am satisfied with the amount of time it took to complete this task and 3 - Overall, I am satisfied with the support information (on-line help, messages, documentation) when completing this task. The ASQ score is obtained as the arithmetic mean of the three answers.

Eye-tracking and mouse data collected from all the participants were used to obtain the transition matrix between AOIs. Data obtained from Tobii Pro Studio for each participant were analyzed to calculate the percentage dwell time (PDT) for each AOI and to obtain the transitions of gaze between the AOIs of the CP. Only dwell times over 300 milliseconds were considered to calculate the transitions between AOIs. Accordingly, short gaze fixations on AOIs while participants are searching for the appropriate next element of the CP were not considered as transitions.

On the other hand, mouse-click events were analyzed registering how many times each AOI was clicked. Comparing eye-tracking and mouse-click events, the cases in which the participants clicked on an AOI while looking at another one were registered. Figure 3 shows the eye-tracking heat map and the mouse tracking (Blascheck et al., 2014) of one participant. This map shows the aggregation of the gaze fixations over time, the mouse path (white lines) and the mouse clicks (yellow circles). The results of these analyses were used to build the transition matrix. The average number of transitions between AOIs for all the participants were calculated (Table 2). These values include the gaze-gaze and the gaze-mouse transitions. The gaze-mouse transitions are shown in brackets in Table 2.

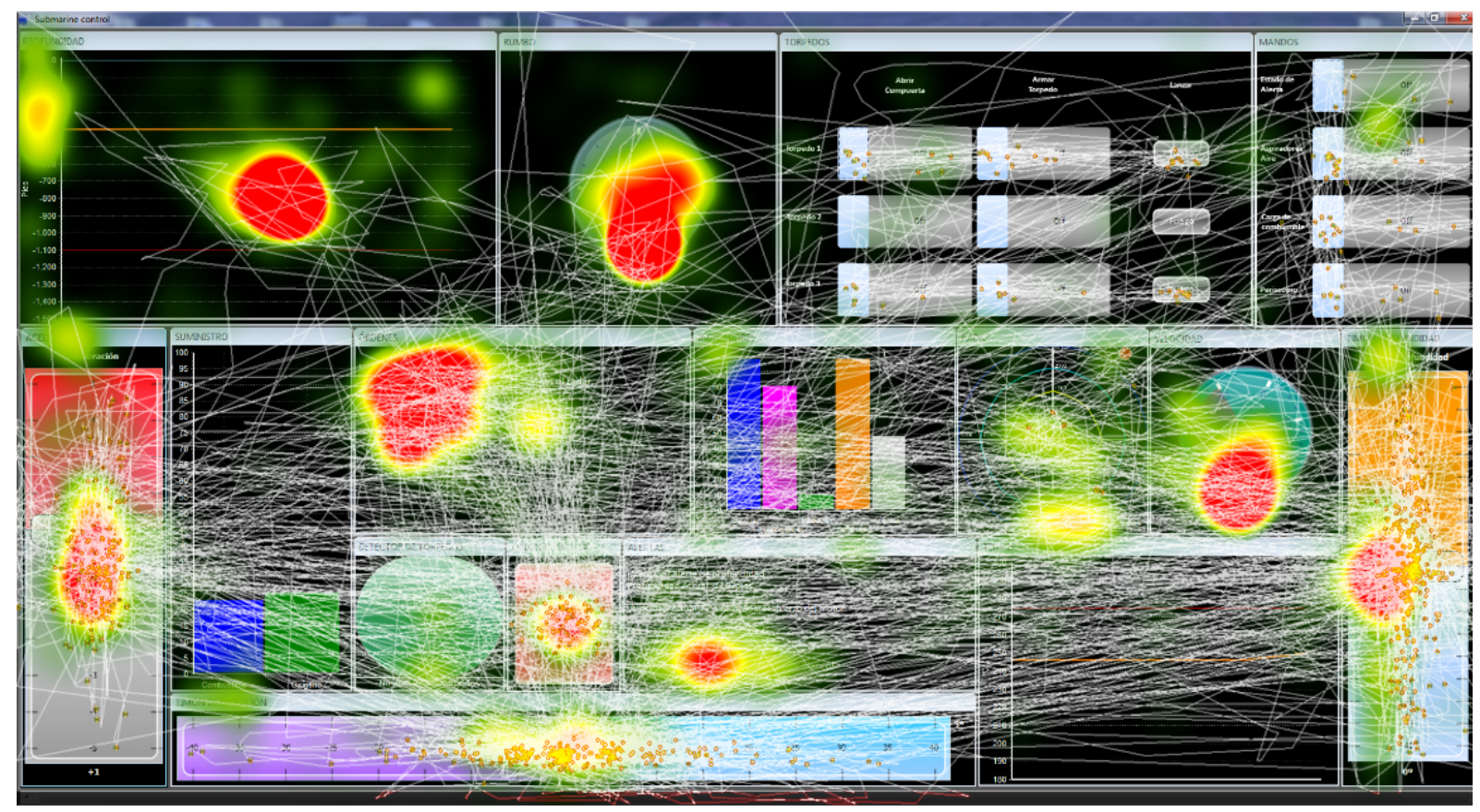

Figure 3: Eye-tracking heat map and mouse path of one participant. The red color represents areas with a large number of gaze fixations over time. The white line is the path of the mouse and yellow circles are the mouse clicks.

The minimum and maximum areas and ratios for each element of the CP shown in Table 1, and the transition matrix between AOIs (Table 2), were used in a slicing-trees based GA (Diego-Mas et al., 2009) to obtain an 
optimal distribution of the elements. Equation1 represents the fitness function of the GA. In this equation, $\mathbf{n}$ was 16 (the number of elements in the CP) and the values established for the relational cost (Rc) and geometrical cost (Gc) were 1 and 2 respectively. The GA used is an algorithm in two stages and both stages were run for 1000 generations. Crossover probability was set at 0.9 and mutation probability at 0.01 . The population size was 50 . The time taken by the calculation process was 4 minutes and 58 seconds on a PC with 12 GB RAM and 3.6 GHz processor.

AOI-1 AOI-2 AOI-3 AOI-4 AOI-5 AOI-6 AOI-7 AOI-8 AOI-9 AOI-10 AOI-11 AOI-12 AOI-13 AOI-14 AOI-A AOI-B

\begin{tabular}{|c|c|c|c|c|c|c|c|c|c|c|c|c|c|c|c|}
\hline AOI-1 & & & & & & & & & & & & & & & \\
\hline AOI-2 & 1.58 & & & & & & & & & & & & & & \\
\hline AOI-3 & 2.46 & 4.00 & & & & & & & & & & & & & \\
\hline AOI-4 & 2.31 & 4.12 & 10.38 & & & & & & & & & & & & \\
\hline AOI-5 & 2.46 & 7.38 & 7.96 & 6.58 & & & & & & & & & & & \\
\hline AOI-6 & 2.46 & 4.35 & 8.50 & 15.31 & 10.69 & & & & & & & & & & \\
\hline AOI-7 & 2.46 & 1.42 & 1.77 & 4.15 & 2.69 & 2.92 & & & & & & & & & \\
\hline AOI-8 & 0.38 & 2.85 & 5.31 & 3.15 & 5.46 & 3.81 & 0.96 & & & & & & & & \\
\hline AOI-9 & 1.27 & 1.42 & 5.81 & 9.00 & 14.46 & 10.08 & 2.46 & 2.73 & & & & & & & \\
\hline AOI-10 & 0.54 & 1.46 & $\begin{array}{c}2.92 \\
(0.10)\end{array}$ & $\begin{array}{c}3.58 \\
(0.05)\end{array}$ & 2.04 & 1.50 & 0.92 & 0.38 & 0.65 & & & & & & \\
\hline AOI-11 & 2.92 & 1.54 & $\begin{array}{c}6.85 \\
(0.25)\end{array}$ & $\begin{array}{c}4.54 \\
(1.25)\end{array}$ & \begin{tabular}{|c|}
29.81 \\
$(15.80)$
\end{tabular} & $\begin{array}{c}97.42 \\
(49.35)\end{array}$ & 2.50 & 3.35 & \begin{tabular}{|c|}
3.46 \\
$(1.05)$
\end{tabular} & 0.15 & & & & & \\
\hline AOI-12 & 0.54 & 2.58 & 3.96 & $\begin{array}{l}104.65 \\
(23.10)\end{array}$ & \begin{tabular}{|l|}
14.31 \\
$(4.09)$ \\
\end{tabular} & \begin{tabular}{|c|}
3.88 \\
$(0.25)$ \\
\end{tabular} & 0.77 & 3.65 & \begin{tabular}{|c|}
7.65 \\
$(0.65)$
\end{tabular} & 2.58 & 7.85 & & & & \\
\hline AOI-13 & $\begin{array}{c}1.19 \\
(0.10)\end{array}$ & 2.58 & $\begin{array}{c}3.85 \\
(1.25)\end{array}$ & $\begin{array}{c}5.88 \\
(1.50)\end{array}$ & \begin{tabular}{|c|}
5.96 \\
$(2.85)$ \\
\end{tabular} & 8.69 & 1.12 & 0.62 & 2.54 & 7.04 & 1.12 & 1.77 & & & \\
\hline AOI-14 & 1.04 & 2.12 & 4.15 & $\begin{array}{c}8.50 \\
(1.05)\end{array}$ & \begin{tabular}{|l|}
18.85 \\
$(4.85)$ \\
\end{tabular} & 5.54 & 3.46 & $\begin{array}{c}3.23 \\
(1.25) \\
\end{array}$ & \begin{tabular}{|c|c}
47.23 \\
$(20.05)$ \\
\end{tabular} & 0.54 & 4.73 & 5.04 & 1.73 & & \\
\hline AOI-A & 4.92 & 6.58 & 23.04 & 47.12 & 13.92 & 32.96 & 6.08 & 3.46 & 17.31 & 11.65 & 14.42 & 17.58 & 24.73 & 16.81 & \\
\hline AOI-B & 4.92 & $\begin{array}{c}2.88 \\
(0.25)\end{array}$ & $\begin{array}{c}9.88 \\
(0.05)\end{array}$ & 8.38 & 4.35 & 8.42 & 6.27 & 2.88 & 3.62 & 2.08 & 3.42 & 2.54 & 3.69 & 7.35 & 36.65 \\
\hline
\end{tabular}

Table 2. Transition matrix of the CP used in the experimental sessions. Each cell represents the average number of gaze-gaze and gaze-mouse transitions between AOIs. Gaze-mouse transitions are shown in brackets.

\subsection{Second experimental session}

In the second experimental session, the participants were randomly separated in two groups of 10 members (group A and group B). The members of the group A were asked to rearrange manually the distribution of the child windows containing the elements of the CP. They had to consider their experience in the first experiment to adapt the layout to their preferences and needs changing the position and size of the elements. Then, they were asked to perform again the same tasks using the new distribution. On the other hand, the members of the group B performed the same sequence using the best layout obtained by the algorithm (Figure 4). Again, the time to complete each task, eye-tracking data and mouse events were collected from all the participants, and all of them completed the ASQ. 

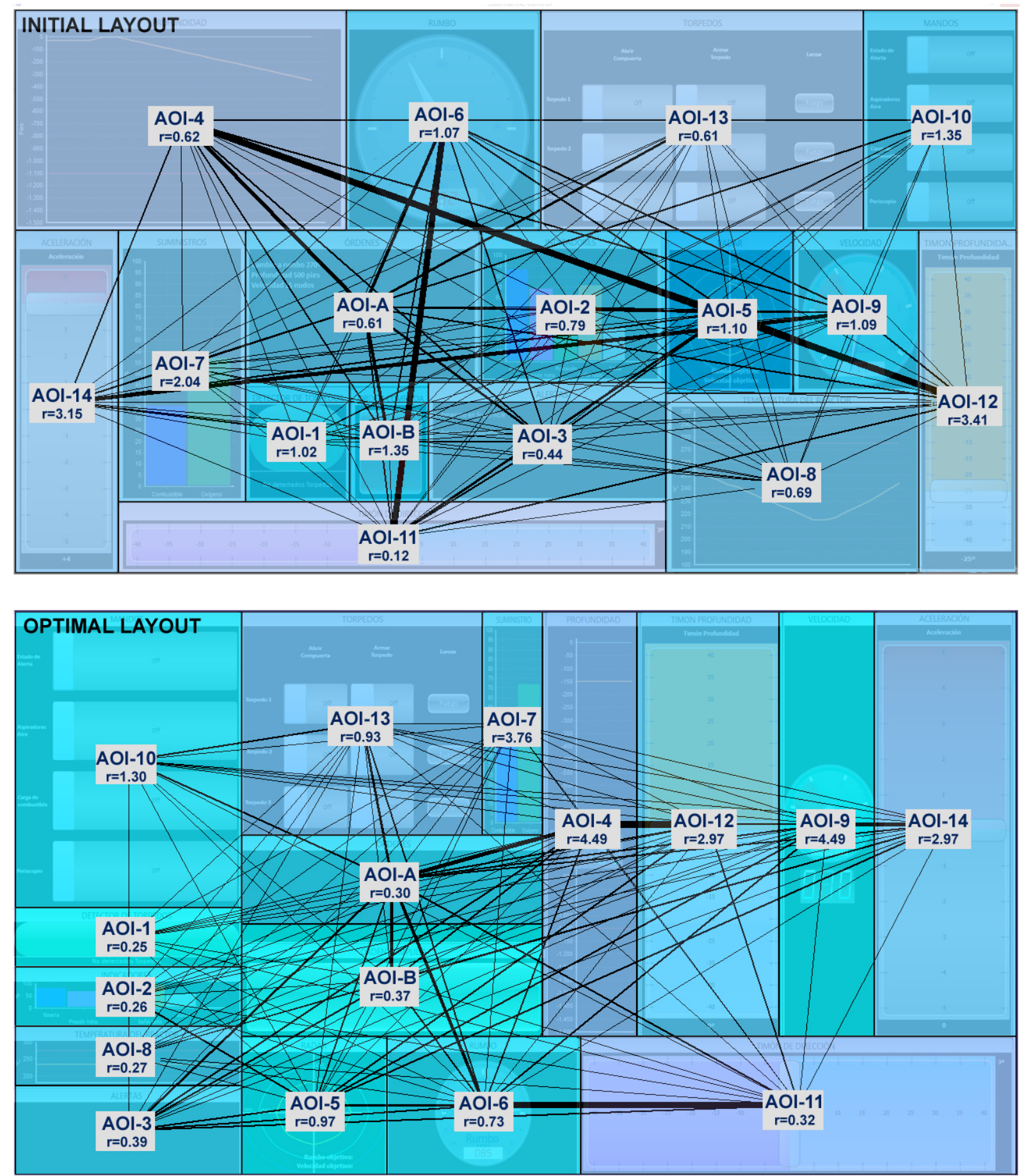

Figure 4: Initial CP layout and optimal layout obtained by the genetic algorithm. Height-width ratios (r) are shown for each element of the control panel.

\section{Results}

Figure 4 shows the initial layout of the CP and the best layout obtained by the GA. In this figure black lines represents the intensity of the relationships between the elements of the $\mathrm{CP}$ as expressed in the transition matrix (Table 2). The greater the thickness of the line between the AOIs, the greater the relationship between them. In the optimal solution, the elements with intense relationships are adjacent or closer than in the initial layout, e.g., 
AOI-4 and AOI-12. The aspect ratio of the elements (r) are also shown in the Figure 4. The aspect ratios of all the elements are between the minimum and maximum ratios (Table 2). Therefore, geometric constraints of all items are met and the geometrical cost (Equation 1) of the solution is 0 , so that the overall cost of the solution is due to the relational cost. The relational costs of the layouts calculated using Equation 1 are 5619.6 for the initial layout and 3798.0 for the optimized layout; therefore, the GA solution achieved a reduction of $32.6 \%$ in the relational cost.

Table A1 in Appendix A section presents the complete results by user for both experimental sessions. Figure 5 presents the data aggregated by experimental session, showing the mean values of the tasks total duration, the errors committed, the ASQ score, the number of mouse clicks and the mouse path length for both experimental groups. Two classes of errors were considered to calculate the number of errors committed by the participants during the experiment. The first class error was not achieving the goal of one task of the predefined sequence of tasks. The second class consisted of initially using an inadequate control to perform a task, although the objective of the task was finally achieved.

Mean values of the usability indicators by experimental session

Tasks duration

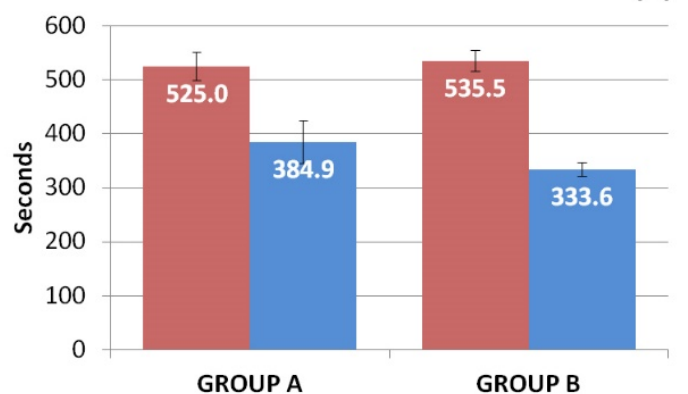

ASQ score

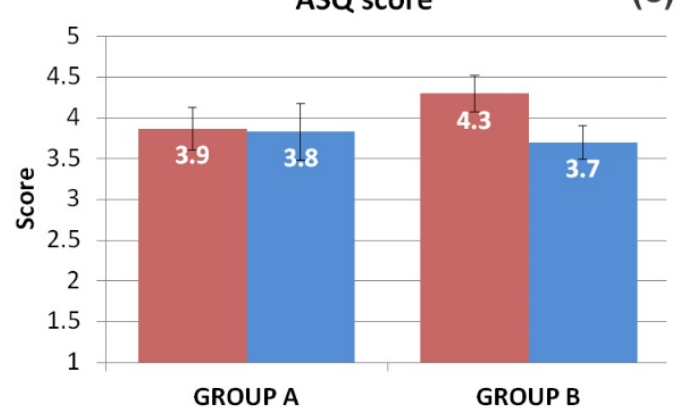

GROUP A

(a)

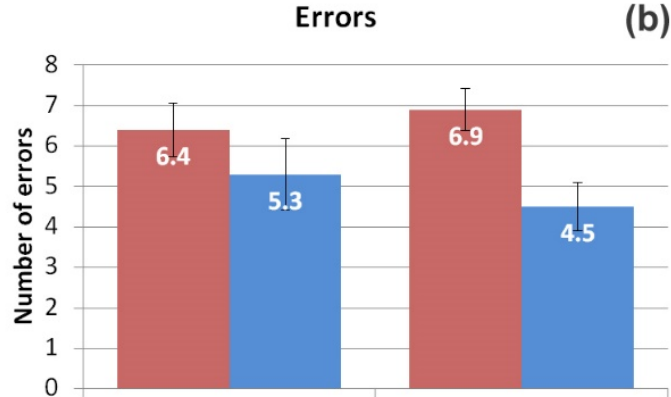

GROUP A

Mouse clicks

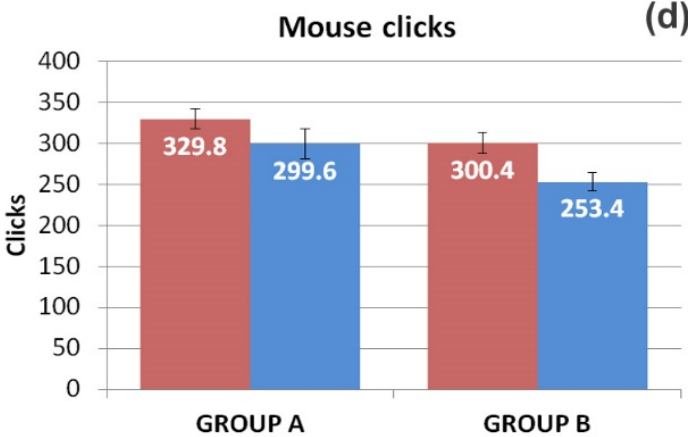

(b)

(d)

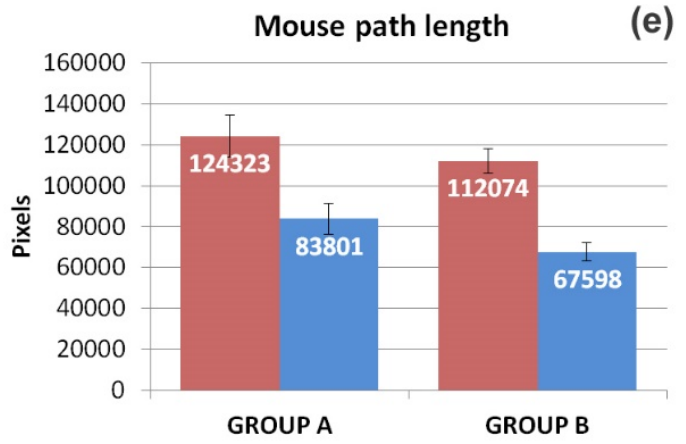

(e)

Session 1

Session 2

Figure 5: Results of the experimental sessions. Bars show, for both experimental groups, the mean values by experimental session of the tasks total duration (a), the errors committed (b), the ASQ score (c), the number of mouse clicks (d) and the mouse path length (e). Whiskers indicate the standard errors of the means. 
Dependent-samples t-tests $(\alpha=0.05)$ were conducted to compare the indicators between sessions. Table A2 in Appendix A section shows the mean and standard deviation of the values of the indicators by experimental session and group, and the t-values and the percentage change in indicators by groups.

Considering all the participants in both experimental sessions, there were significant differences in all the indicators between sessions. By groups, the mean value of the task duration decreased by $26.70 \%$ for Group A and $37.70 \%$ for Group B. The difference in the reduction of the number of errors was greater; while the participants of the Group A reduced the errors by 17.19\%, those of Group B decreased the number of errors committed by $34.78 \%$. On the other hand, there was a slight reduction in the ASQ score between sessions for the Group A (1.03\%), but this difference was not significant. The ASQ score of the participants of the Group B decreased by 13.95\%. Because of the Likert scale used in this questionnaire, lower values of the scores correspond to higher levels of satisfaction. The number of mouse clicks needed to perform the tasks diminished by $9.16 \%$ in the Group A, and by $15.65 \%$ in the Group B, and the mouse trail length decreased by $32.59 \%$ in the Group A, and by 39.69\% in the Group B.

Finally, Pearson product-moment correlation coefficients were calculated to measure the relationship between all the indicators between sessions for all the participants. There were positive correlations between the task duration $(r=0.506, n=20, p=0.023)$, number of errors $(r=0.857, n=20, p=0.000)$, ASQ scores $(r=0.767, n$ $=20, p=0.000)$ and number of mouse clicks $(r=0.691, n=20, p=0.001)$, for all the participants between sessions. Low and not significant correlation was found for the mouse path length between sessions $(r=0.117, n$ $=20, \mathrm{p}=0.625$ ). Positive significant correlations were also found between the ASQ scores and the task duration in both sessions ( $r=0.5443, \mathrm{n}=20, \mathrm{p}=0.013$ for session $1 ; \mathrm{r}=0.5391, \mathrm{n}=20, \mathrm{p}=0.014$ for session 2 ), and between the ASQ scores and the number of errors $(r=0.6995, n=20, p=0.001$ for session $1 ; r=0.7985, n=20$, $\mathrm{p}=0.000$ for session 2).

\section{Discussion}

The main objective of the redesign of a user interface is to improve its usability. ISO 9241-11 standard (International Organization for Standardization, 1998) defines usability as: "the extent to which a product can be used by specified users to achieve specified goals with effectiveness, efficiency and satisfaction in a specified context of use". Three different aspects are involved in this definition: effectiveness, efficiency and satisfaction. Each of them must be measured using different indicators (Frøkjær et al., 2000). Error rates is a good indicator of effectiveness, indicators of efficiency include task completion time and attitude-rating scales can measure users' satisfaction.

We used error rates as indicator of effectiveness and the length of the mouse trail and the number of mouse clicks that the users need to perform the tasks in the CP as indicator of efficiency. Intuitively, both of them are related to the amount of effort required to achieve the proposed goals. The longer the mouse trail and the higher the number of mouse clicks, the more choices and relevance judgments are needed to be made by the operator (Gwizdka and Spence, 2007). On the other hand, more mouse clicks could occur when the operator uses successively a control to correct a previous mistake or when he finds difficulties to use it properly. These relationships have been proved in the context of web searching (Cox and Silva, 2006; Huang et al., 2011; Kim, 
2006; Mueller and Lockerd, 2001), however, to the best of our knowledge, they must still be tested for other kinds of interfaces.

We used the ASQ (Lewis, 1995) to measure the users' satisfaction. Common usability and user experience surveys (Tullis and Albert, 2013) evaluate multiple aspects of the interfaces as sequence of screens, terminology, readability, help and documentation, interface learnability, etc. The procedure presented in this work is intended to obtain optimal layouts of the elements of an interface without modifying their characteristics (except their position and, to a lesser extent, their size). The kind of control and indicators or other features as functionality or colors are not modified by this system. ASQ is a simple questionnaire intended to determining the user satisfaction in scenario-based usability studies. Therefore, we selected it to measure the users' satisfaction.

The experiment performed in this work has shown that the GA used in the proposed system is able to find solutions that considers the geometrical restrictions of the elements of the interface. As all the geometric constraints are met, the solution found has no geometric cost, and the overall cost is due to the relational cost. Therefore, the optimized layout obtained can be directly implanted without needing manual adjustments achieving a reduction of $32.6 \%$ in the relational cost.

We performed two experimental sessions with two groups (A and B) of 10 users. All 20 users participated in the first experimental session completing a sequence of 20 tasks on a CP arranged as in Figure 1a. In the second experimental session, the members of the group A were asked to rearrange manually the distribution of the CP considering their experiences in the first session and to perform the same sequence of tasks. The members of the group B used the best layout obtained by the algorithm (Figure 4). The time to tasks completion, errors made, eye-tracking data and mouse events were collected from all the participants, and all of them completed the ASQ.

Figure 6 shows the percentage change in usability indicators between the sessions. The participants were more familiar with the interface in the second session. Therefore, as it might be expected, all the usability indicators decreased in the second experimental session. Because of their definitions, lower values of the usability indicators correspond to higher levels of usability. The decrease was more pronounced for the participants that used the optimized CP (Group B) than for those that used their own arrangement (Group A). Dependent-samples t-tests $(\alpha=0.05)$ conducted showed that the differences between sessions were significant except the slight reduction in the ASQ score for the Group A.

These results suggest that the CP layout obtained using the procedure proposed in this work increases effectiveness, efficiency and user satisfaction compared to user self-defined arrangements. However, the obtained values must be compared between groups before concluding that the improvement is due to different layouts. Different skills, abilities or capacities of the participants in each group might as well explain this fact. The independent t-tests $(\alpha=0.05)$ conducted to compare the indicators between groups (Table A3) show that there were not significantly different indicators values between groups for the experimental session 1 , when all the participants used the same interface, being the values for task duration, errors and ASQ score lower for Group A. Conversely, the mean values of the indicators were higher for the group using the optimized interface in session 2. Therefore, it could be concluded that the differences in effectiveness, efficiency and user satisfaction between groups are not due to the different capacities of its members. 


\section{Percentage change in usability indicators between sessions by groups of participants}

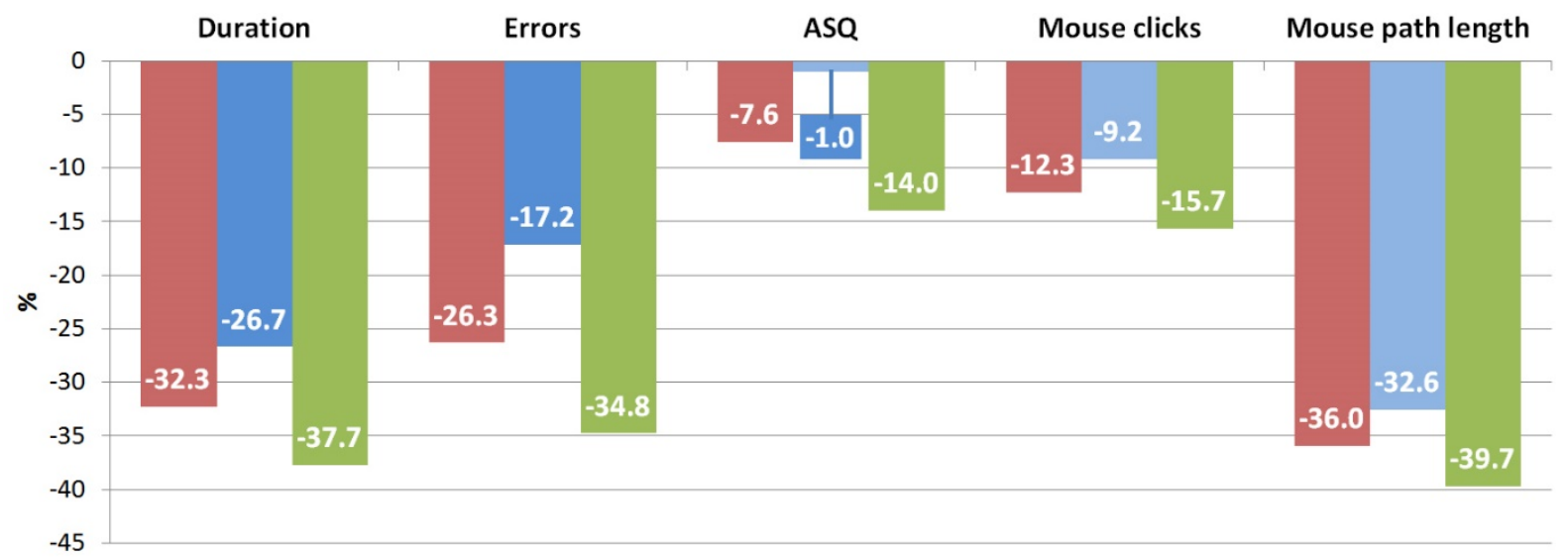

all participants $\|$ Group A $\approx$ Group B

Figure 6: Percentage change in the mean values of the usability indicators between sessions by groups of participants.

Pearson product-moment correlation coefficients were calculated to measure the relationship between all the indicators between sessions for all the participants. Overall, in all indicators except for the mouse path length, as the result obtained in the first session improves so does that in the second. This outcome shows that individual abilities, skills and preferences of the participants significantly affects the effectiveness, efficiency and satisfaction when using the interface regardless of its configuration. On the other hand, the mouse path length by participant does not show correlation between sessions. While the users of the interface are looking for the appropriate control or indicator, mouse cursor assist them with visual search using the pointer as a marker (Cox and Silva, 2006). Figure 7 shows the mouse trail for one participant for the sessions. Although this aspect needs more specific research, the length of the mouse path could be an indicator of the familiarity with the task to be performed using the CP (Todi et al., 2018), and its variation between sessions seems to be independent of the user ability or skills. This is consistent with some previous studies about web searching (Huang et al., 2011; Liu et al., 2010; Mueller and Lockerd, 2001).

The procedure proposed in this work is intended to optimize the layout of software-based interfaces such as control panels or applications with customizable menus and toolbars in order to improve their usability. Our proposal uses Eye-tracking and mouse-tracking, non-invasive techniques that permit gathering the data required by the GA to obtain new layouts while the operators perform their usual tasks. Therefore, the system can be used to generate static or adaptive user interfaces (Gajos et al., 2006a; Todi et al., 2018). Static user interfaces remain unchanged once designed and implemented. The data to optimize static interfaces can be gathered from users during a time interval of normal operation of the CP or during controlled experimental sessions. On the other hand, adaptive user interfaces can adapt or modify themselves while being used based on users' interaction history. Our system can propose new optimized arrangements in an automatized way based on data collected permanently from the users during the normal operation of the CP. 

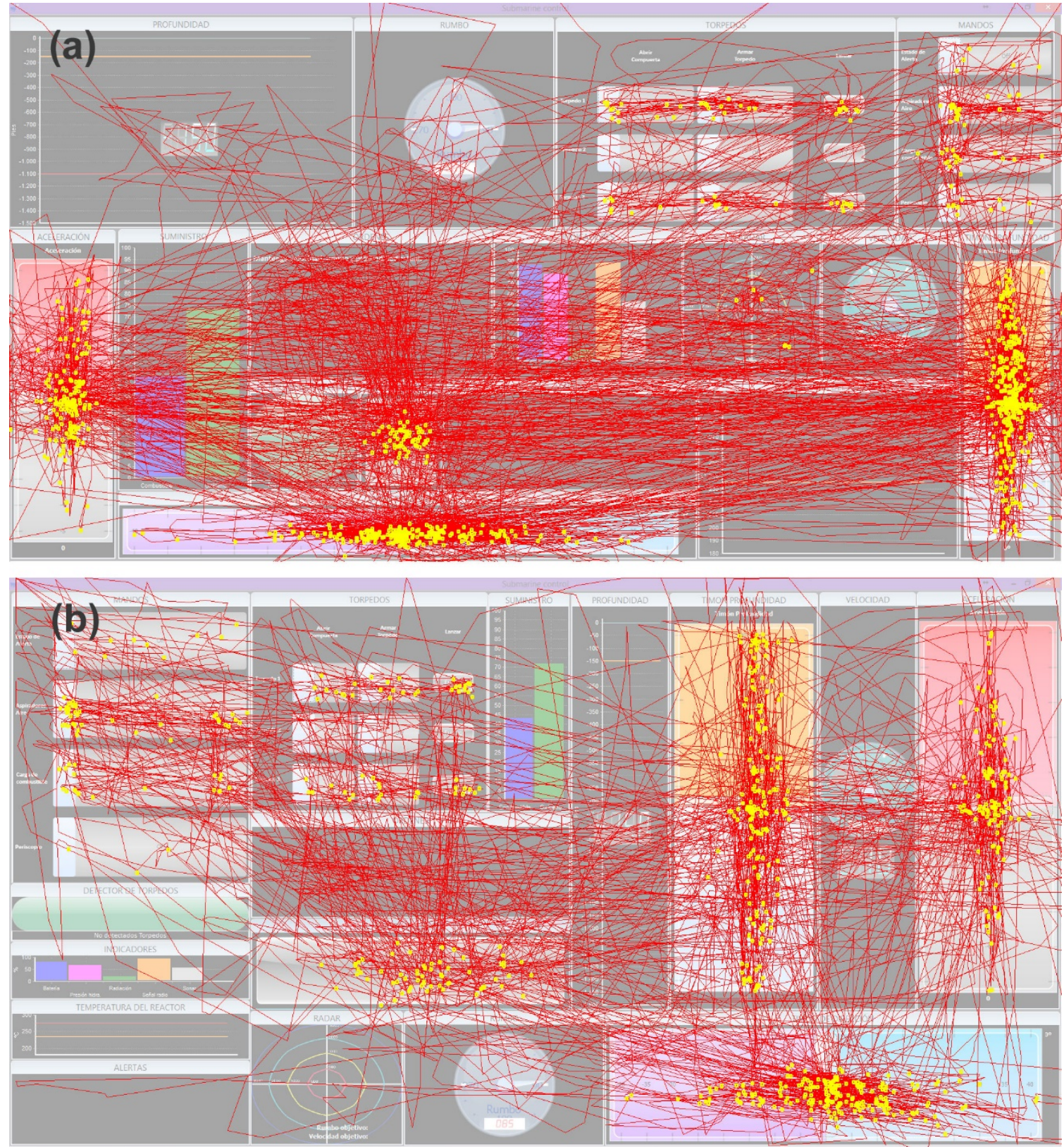

Figure 7: Mouse path (red lines) and clicks (yellow points) of a participant of the Group B in both experimental sessions. (a) Experimental session 1 with the initial layout of the CP. (b) Experimental session 2 with the final layout of the CP obtained by the genetic algorithm.

Furthermore, it is possible to use data collected from individual users to find per-user customized interfaces, or data from several users to obtain a common arrangement optimized for several operators. Using custom user interfaces for each operator takes into account the abilities and requirements of specific users (Gajos et al., 2006b; Gamecho et al., 2015; Nichols et al., 2006; Nichols and Lau, 2008). In the same way, it is possible to obtain pertask customized interfaces using the data gathered while the users are performing specific tasks (Gajos et al., 2006a).

Some limitations of the proposed procedure must be pointed out. The current approach makes use of the intensity of the relationship between two elements of a $\mathrm{CP}$ and the distance between them in the layout to calculate the relational cost that the GA must minimize. The intensity of the relationship between two elements of a CP is 
measured using the number of transitions between the elements. We have considered two kinds of transitions: gaze-gaze transitions and gaze-mouse transitions. Currently, both kinds of transitions are equally weighted in the fitness function, giving them the same importance in obtaining the optimal layout. Further research must study if using different weighting coefficients for each of them may lead to improve the usability of the obtained CP, and how it affects to effectiveness, efficiency and users' satisfaction.

\section{Conclusions}

In this work we have used eye-tracking and mouse-tracking data for optimize the layout of the elements of a CP using a slicing trees based GA. The algorithm used considers the geometrical restrictions of the elements of the interface during the search for the optimal layout. Consequently, the obtained solution can be directly implanted without the need for manual adjustments that distort the layout. The results of our experiments show that the layouts obtained using this procedure outperforms user-defined interfaces arrangements in effectiveness, efficiency and user satisfaction.

\section{Appendix A}

\section{Results of the experimental sessions.}

\begin{tabular}{ccccccccccccc}
\hline & & \multicolumn{2}{c}{ Duration (s) } & \multicolumn{2}{c}{ Errors } & \multicolumn{2}{c}{ ASQ } & \multicolumn{2}{c}{ Mouse Clicks } & \multicolumn{2}{c}{ Mouse path length } \\
\cline { 3 - 12 } GROUP & USER & S1 & S2 & S1 & S2 & S1 & S2 & S1 & S2 & S1 & S2 \\
\hline A & U1 & 542.14 & 370.44 & 7 & 6 & 4.00 & 4.00 & 332 & 300 & 127546 & 82343 \\
A & U2 & 560.43 & 377.60 & 5 & 6 & 3.67 & 4.00 & 346 & 315 & 124469 & 84769 \\
A & U3 & 471.08 & 378.61 & 3 & 3 & 2.67 & 3.00 & 267 & 301 & 105581 & 86024 \\
A & U4 & 497.84 & 371.08 & 7 & 5 & 3.67 & 3.00 & 336 & 278 & 104843 & 79863 \\
A & U5 & 465.96 & 372.28 & 4 & 3 & 3.33 & 3.67 & 365 & 388 & 110071 & 117162 \\
A & U6 & 386.34 & 279.66 & 6 & 4 & 3.33 & 3.00 & 257 & 228 & 69834 & 58079 \\
A & U7 & 622.45 & 326.41 & 7 & 6 & 4.67 & 4.00 & 350 & 305 & 184256 & 54653 \\
A & U8 & 617.82 & 498.54 & 9 & 8 & 5.67 & 5.33 & 354 & 308 & 167325 & 87956 \\
A & U9 & 463.77 & 428.94 & 10 & 7 & 4.00 & 4.33 & 369 & 262 & 118588 & 109333 \\
A & U10 & 622.66 & 445.15 & 6 & 5 & 3.67 & 4.00 & 322 & 311 & 130716 & 77826 \\
B & U11 & 555.94 & 301.74 & 9 & 6 & 4.00 & 3.67 & 255 & 226 & 97222 & 75854 \\
B & U12 & 548.85 & 304.14 & 5 & 4 & 4.33 & 4.00 & 290 & 251 & 150469 & 62385 \\
B & U13 & 566.96 & 382.64 & 3 & 1 & 3.33 & 3.00 & 279 & 259 & 85659 & 47890 \\
B & U14 & 558.67 & 352.61 & 3 & 2 & 3.00 & 3.00 & 335 & 275 & 100197 & 69994 \\
B & U15 & 537.46 & 377.84 & 11 & 7 & 6.00 & 5.00 & 401 & 340 & 103251 & 55489 \\
B & U16 & 309.68 & 272.24 & 8 & 6 & 4.00 & 3.67 & 220 & 215 & 86557 & 83408 \\
B & U17 & 747.14 & 382.42 & 8 & 5 & 6.00 & 4.00 & 374 & 252 & 149917 & 93349 \\
B & U18 & 681.74 & 359.04 & 10 & 6 & 5.00 & 4.33 & 256 & 238 & 100040 & 58339 \\
B & U19 & 412.02 & 296.47 & 7 & 4 & 3.00 & 3.33 & 266 & 227 & 128072 & 73192 \\
B & U20 & 436.49 & 306.96 & 5 & 4 & 4.33 & 3.00 & 328 & 251 & 119357 & 56075 \\
\hline
\end{tabular}

Table A1. Results of the experimental sessions. Task duration (seconds), errors committed, After Scenario Questionnaire score (ASQ), number of mouse clicks and mouse path length (in pixels) for both experimental sessions (S1 and S2) are shown by user. 


\begin{tabular}{|c|c|c|c|c|c|c|c|}
\hline & \multirow[b]{2}{*}{ Indicator } & \multicolumn{2}{|c|}{ Session 1} & \multicolumn{2}{|c|}{ Session 2} & \multirow[b]{2}{*}{ t-test } & \multirow[b]{2}{*}{$\% \mathrm{M}$} \\
\hline & & M & SD & M & SD & & \\
\hline \multirow{5}{*}{$\begin{array}{l}\text { All } \\
\text { participants }\end{array}$} & Duration (s) & 530.27 & 103.67 & 359.24 & 57.24 & $8.54^{* *}$ & -32.25 \\
\hline & Errors & 6.65 & 2.43 & 4.90 & 1.77 & $6.05^{* *}$ & -26.32 \\
\hline & ASQ & 4.08 & 0.97 & 3.77 & 0.68 & $2.27 * *$ & -7.60 \\
\hline & Mouse clicks & 315.10 & 49.97 & 276.50 & 44.21 & $4.62 * *$ & -12.25 \\
\hline & Mouse path length & 118198.54 & 28402.00 & 75699.13 & 18445.20 & $5.94 * *$ & -35.96 \\
\hline \multirow{5}{*}{ Group A } & Duration (s) & 525.05 & 81.18 & 384.87 & 61.13 & $6.22 * *$ & -26.70 \\
\hline & Errors & 6.40 & 2.12 & 5.30 & 1.64 & $3.16^{* *}$ & -17.19 \\
\hline & ASQ & 3.87 & 0.82 & 3.83 & 0.72 & 0.26 & -1.03 \\
\hline & Mouse clicks & 329.80 & 38.56 & 299.60 & 41.22 & $2.38 * *$ & -9.16 \\
\hline & Mouse path length & 124322.96 & 32366.30 & 83800.79 & 19300.40 & $3.21^{* *}$ & -32.59 \\
\hline \multirow{5}{*}{ Group B } & Duration (s) & 535.50 & 126.64 & 333.61 & 41.49 & $6.47^{* *}$ & -37.70 \\
\hline & Errors & 6.90 & 2.81 & 4.50 & 1.90 & $6.47 * *$ & -34.78 \\
\hline & ASQ & 4.30 & 1.09 & 3.70 & 0.66 & $2.78^{* *}$ & -13.95 \\
\hline & Mouse clicks & 300.40 & 57.49 & 253.40 & 35.23 & $4.32 * *$ & -15.65 \\
\hline & Mouse path length & 112074.12 & 23917.50 & 67597.47 & 14138.10 & $5.96^{* *}$ & -39.69 \\
\hline
\end{tabular}

${ }^{* *} \mathrm{p}<0.05 . \mathrm{M}=$ Mean. $\mathrm{SD}=$ Standard deviation. $\% \mathrm{M}=$ Mean percent variation between sessions.

Table A2. Mean and standard deviation of the values of the indicators by group and session, and t-values for the dependent-samples t-tests between sessions conducted.

\begin{tabular}{|c|c|c|c|c|c|c|c|}
\hline & \multirow[b]{2}{*}{ Indicator } & \multicolumn{2}{|c|}{ Group A } & \multicolumn{2}{|c|}{ Group B } & \multirow[b]{2}{*}{ t-test } & \multirow[b]{2}{*}{$\% \mathrm{M}$} \\
\hline & & M & SD & $M$ & SD & & \\
\hline \multirow{5}{*}{ Session 1} & Duration (s) & 525.05 & 81.18 & 535.50 & 126.64 & -0.22 & -1.99 \\
\hline & Errors & 6.40 & 2.12 & 6.90 & 2.81 & -0.45 & -7.81 \\
\hline & ASQ & 3.87 & 0.82 & 4.30 & 1.09 & -0.99 & -11.11 \\
\hline & Mouse clicks & 329.80 & 38.56 & 300.40 & 57.49 & 1.34 & 8.91 \\
\hline & Mouse path length & 124322.96 & 32366.30 & 112074.12 & 23917.50 & 0.96 & 9.85 \\
\hline \multirow{5}{*}{ Session 2} & Duration (s) & 384.87 & 61.13 & 333.61 & 41.49 & $2.19 * *$ & 13.32 \\
\hline & Errors & 5.30 & 1.64 & 4.50 & 1.90 & 1.01 & 15.09 \\
\hline & ASQ & 3.83 & 0.72 & 3.70 & 0.66 & 0.43 & 3.39 \\
\hline & Mouse clicks & 299.60 & 41.22 & 253.40 & 35.23 & $2.69 * *$ & 15.42 \\
\hline & Mouse path length & 83800.79 & 19300.40 & 67597.47 & 14138.10 & $2.14^{* *}$ & 19.34 \\
\hline
\end{tabular}

${ }^{* *} \mathrm{p}<0.05 . \mathrm{M}=$ Mean. SD = Standard deviation. \%M = Mean percent variation between groups.

Table A3. Mean and standard deviation of the values of the indicators by session and group, and t-values for the independent-samples t-tests between groups conducted.

\section{Acknowledgements}

This work was supported by the Programa estatal de investigación, desarrollo e innovación orientada a los retos de la sociedad of the Government of Spain under Grant DPI2016-79042-R. 


\section{References}

Alluisi, E.A., Morgan, B.B., 1976. Engineering Psychology and Human Performance. Annu. Rev. Psychol. 27, 305-330. doi:10.1146/annurev.ps.27.020176.001513

Alppay, C., Bayazit, N., 2015. An ergonomics based design research method for the arrangement of helicopter flight instrument panels. Appl. Ergon. 51, 85-101. doi:10.1016/j.apergo.2015.04.011

Blascheck, T., Kurzhals, K., Raschke, M., Burch, M., Weiskopf, D., Ertl, T., 2014. State-of-the-Art of Visualization for Eye Tracking Data. Eurographics Conf. Vis. 1-20. doi:10.2312/eurovisstar.20141173

Cai, H., Liu, B., Zhang, J., Chen, S., Liu, H., 2017. Visual focus of attention estimation using eye center localization. IEEE Syst. J. 11, 1320-1325. doi:10.1109/JSYST.2015.2441952

Carvalho, P.V.R., dos Santos, I.L., Gomes, J.O., Borges, M.R.S., Guerlain, S., 2008. Human factors approach for evaluation and redesign of human-system interfaces of a nuclear power plant simulator. Displays 29, 273284. doi:10.1016/j.displa.2007.08.010

Chapanis, A., 1996. Human factors in systems engineering. John Wiley \& Sons, Inc, New York, NY.

Cox, A.L., Silva, M.M., 2006. The Role of Mouse Movements in Interactive Search. Proc. Cogn. Sci. Soc. 28.

Degani, A., Palmer, E.A., Bauersfeld, K.G., 1992. “Soft” Controls for hard displays: still a challenge. Proc. Hum. Factors Ergon. Soc. Annu. Meet. 36, 52-56. doi:10.1177/154193129203600114

Diego-Mas, J.A., Poveda-Bautista, R., Garzon-Leal, D., 2017. Using RGB-D sensors and evolutionary algorithms for the optimization of workstation layouts. Appl. Ergon. 65, 530-540. doi:10.1016/j.apergo.2017.01.012

Diego-Mas, J.A., Santamarina-Siurana, M.C., Alcaide-Marzal, J., Cloquell-Ballester, V.A., 2009. Solving facility layout problems with strict geometric constraints using a two-phase genetic algorithm. Int. J. Prod. Res. 47, 1679-1693. doi:http://dx.doi.org/10.1080/00207540701666253">http://dx.doi.org/10.1080/00207540701666253

Diego-Mas, J.A., Santamarina-Siurana, M.C., Cloquell-Ballester, V.A., Alcaide-Marzal, J., 2008. Slicing tree’s geometric potential: an indicator for layout problems based on slicing tree structure. Int. J. Prod. Res. 46, 1071-1087. doi:10.1080/00207540600932061

El-Baz, M.A., 2004. A genetic algorithm for facility layout problems of different manufacturing environments. Comput. Ind. Eng. 47, 233-246. doi:10.1016/j.cie.2004.07.001

Foley, J.D., Kim, W.C., Kovacevic, S., Murray, K., 1991. UIDE - An intelligent user interface design environment, in: Architectures for Intelligent Interfaces: Elements and Prototypes. pp. 339-384. doi:10.1145/107215.128716

Francis, G., 2000. Designing Multifunction Displays: An Optimization Approach. Int. J. Cogn. Ergon. 4, 107124. doi:10.1207/S15327566IJCE0402_2 
Frøkjær, E., Hertzum, M., Hornbæk, K., 2000. Measuring Usability : Are Effectiveness , Efficiency , and Satisfaction Really Correlated? ACM CHI 2000 Conf. Hum. Factors Comput. Syst. 2, 345-352. doi:10.1145/332040.332455

Gajos, K.Z., Czerwinski, M., Tan, D.S., Weld, D.S., 2006a. Exploring the design space for adaptive graphical user interfaces, in: Proceedings of the Working Conference on Advanced Visual Interfaces - AVI ’06. p. 201. doi:10.1145/1133265.1133306

Gajos, K.Z., Long, J.J., Weld, D.S., 2006b. Automatically generating custom user interfaces for users with physical disabilities. Proc. 8th Int. ACM SIGACCESS Conf. Comput. Access. - Assets ’06 243. doi:10.1145/1168987.1169036

Gamecho, B., Minon, R., Aizpurua, A., Cearreta, I., Arrue, M., Garay-Vitoria, N., Abascal, J., 2015. Automatic Generation of Tailored Accessible User Interfaces for Ubiquitous Services. IEEE Trans. Human-Machine Syst. 45, 612-623. doi:10.1109/THMS.2014.2384452

Geman, S., Geman, D., 1984. Stochastic Relaxation, Gibbs Distributions, and the Bayesian Restoration of Images. IEEE Trans. Pattern Anal. Mach. Intell. PAMI-6, 721-741. doi:10.1109/TPAMI.1984.4767596

Goldberg, D.E., 1989. Genetic algorithms in search, optimization and machine learning. Addison-Wesley Longman Publishing Co., Inc, Boston, MA.

Goldberg, J.H., Kotval, X.P., 1999. Computer interface evaluation using eye movements: Methods and constructs. Int. J. Ind. Ergon. 24, 631-645. doi:10.1016/S0169-8141(98)00068-7

Gwizdka, J., Spence, I., 2007. What Can Searching Behavior Tell Us About the Difficulty of Information Tasks? A Study of Web Navigation. Proc. Am. Soc. Inf. Sci. Technol. 43, 1-22. doi:10.1002/meet.14504301167

Harris, R.L., Glover, B.L., Spady, A.A., 1986. Analytic techniques of pilot scanning behavior and their application. (NASA Tech. Rep. No. 2525). Hampton, VA.

Helleberg, J.R., Wickens, C.D., 2003. Effects of data-link modality and display redundancy on pilot performance: An attentional perspective. Int. J. Aviat. Psychol. 13, 189-210. doi:10.1207/S15327108IJAP1303_01

Hessels, R.S., Kemner, C., van den Boomen, C., Hooge, I.T.C., 2016. The area-of-interest problem in eyetracking research: A noise-robust solution for face and sparse stimuli. Behav. Res. Methods 48, 16941712. doi:10.3758/s13428-015-0676-y

Holland, J.H., 1975. Adaptation in Natural and Artificial Systems, University of Michigan Press. Ann Arbor.

Holman, G.T., Carnahan, B.J., Bulfin, R.L., 2003. Using linear programming to optimize control panel design from an ergonomics perspective. Proc. Hum. Factors Ergon. Soc. Annu. Meet. 47, 1317-1321. doi:10.1177/154193120304701047

Holmqvist, K., Nyström, M., Andersson, R., Dewhurst, R., Jarodzka, H., van de Weijer, J., 2011. Eye tracking: A comprehensive guide to methods and measures. Oxford University Press, Oxford. 
Horberry, T.J., Burgess-Limerick, R., Steiner, L.J., 2010. Controls and displays, in: Human Factors for the Design, Operation, and Maintenance of Mining Equipment. pp. 119-144. doi:doi:10.1201/9781439802335-c8

Hosseini-Nasab, H., Emami, L., 2013. A hybrid particle swarm optimisation for dynamic facility layout problem. Int. J. Prod. Res. 51, 4325-4335. doi:10.1080/00207543.2013.774486

Huang, J., White, R.W., Dumais, S., 2011. No clicks, no problem: Using cursor movements to understand and improve search. Proc. 29th SIGCHI Conf. Hum. Factors Comput. Syst. 1225.

doi:10.1145/1978942.1979125

Hwang, S.L., Liang, S.F.M., Liu, T.Y.Y., Yang, Y.J., Chen, P.Y., Chuang, C.F., 2009. Evaluation of human factors in interface design in main control rooms. Nucl. Eng. Des. 239, 3069-3075. doi:10.1016/j.nucengdes.2009.09.006

International Organization for Standardization, 1998. ISO 9241-11: Ergonomic requirements for office work with visual display terminals (VDTs) - part 11: guidance on usability. Int. Organ. Stand. 1998, 28. doi:10.1038/sj.mp.4001776

International Organization for Standardization, 2008. Ergonomic design of control centres — Part 5: Displays and controls.

James, T., Rego, C., Glover, F., 2009. Multistart tabu search and diversification strategies for the quadratic assignment problem. IEEE Trans. Syst. Man, Cybern. Part ASystems Humans 39, 579-596. doi:10.1109/TSMCA.2009.2014556

Kantowitz, B.H., Sorkin, R.D., 1983. Human factors : understanding people-system relationships. Wiley, New York.

Karn, K.S., Ellis, S., Juliano, C., 1999. Embedded eye tracker in a real aircraft: new perspectives on pilot/aircraft interaction monitoring, in: CHI '99 Extended Abstracts on Human Factors in Computing Systems - CHI ’99. p. 173. doi:10.1145/632716.632823

Karrenbauer, A., Oulasvirta, A., 2014. Improvements to Keyboard Optimization with Integer Programming, in: Proceedings of the 27th Annual ACM Symposium on User Interface Software and Technology - UIST '14. pp. 621-626. doi:10.1145/2642918.2647382

Kim, J., 2006. Task difficulty as a predictor and indicator of web searching interaction. Proc. ACM CHI 2006 Conf. Hum. Factors Comput. Syst. 2, 959-964. doi:10.1145/1125451.1125636

Kim, W.C., Foley, J.D., 1990. DON: user interface presentation design assistant, in: UIST. Third Annual Symposium on User Interface Software and Technology. Proceedings of the ACM SIGGRAPH Symposium, Snowbird, Utah, USA, October 3--5, 1990. pp. 10-20. doi:10.1145/97924.97926

Kroemer, K.H.E., Kroemer, H.B., Kroemer-Elbert, K.E., 2001. Ergonomics: How to design for ease and efficiency. Prentice Hall, Upper Saddle River, New Jersey. 
Küçükoğlu, İ., Öztürk, N., 2017. Two-stage optimisation method for material flow and allocation management in cross-docking networks. Int. J. Prod. Res. 55, 410-429. doi:10.1080/00207543.2016.1184346

Kulturel-Konak, S., Konak, A., 2015. A large-scale hybrid simulated annealing algorithm for cyclic facility layout problems. Eng. Optim. 47, 963-978. doi:10.1080/0305215X.2014.933825

Kusiak, A., Heragu, S.S., 1987. The facility layout problem. Eur. J. Oper. Res. doi:10.1016/03772217(87)90238-4

Lewis, J.R., 1995. IBM Computer Usability Satisfaction Questionnaires: Psychometric Evaluation and Instructions for Use. Int. J. Hum. Comput. Interact. 7, 57-78. doi:10.1080/10447319509526110

Lin, C.-J., Wu, C., 2010. Improved link analysis method for user interface design - modified link table and optimisation-based algorithm. Behav. Inf. Technol. 29, 199-216. doi:10.1080/01449290903233892

Liu, J., Gwizdka, J., Liu, C., Belkin, N.J., 2010. Predicting task difficulty for different task types. Proc. Am. Soc. Inf. Sci. Technol. 47, 1-10. doi:10.1002/meet.14504701173

Martin, C., Cegarra, J., Averty, P., 2011. Analysis of Mental Workload during En-route Air Traffic Control Task Execution Based on Eye-Tracking Technique. Springer, Berlin, Heidelberg, pp. 592-597. doi:10.1007/978-3-642-21741-8_63

McKendall, A.R., Liu, W.-H., 2012. New Tabu search heuristics for the dynamic facility layout problem. Int. J. Prod. Res. 50, 867-878. doi:10.1080/00207543.2010.545446

Merz, P., Freisleben, B., 1999. A comparison of memetic algorithms, tabu search, and ant colonies for the quadratic assignment problem. Proc. 1999 Congr. Evol. Comput. Cat No 99TH8406 3, 2063-2070. doi:10.1109/CEC.1999.785529

Mueller, F., Lockerd, A., 2001. Cheese: tracking mouse movement activity on websites, a tool for user modeling. CHI’01 Ext. Abstr. Hum. factors ... 279-280. doi:10.1145/634067.634233

Nachreiner, F., Nickel, P., Meyer, I., 2006. Human factors in process control systems: The design of humanmachine interfaces, in: Safety Science. pp. 5-26. doi:10.1016/j.ssci.2005.09.003

Nichols, J., Lau, T., 2008. Mobilization by demonstration: using traces to re-author existing web sites. Proc. 13th Int. Conf. ... 149-158. doi:10.1145/1378773.1378793

Nichols, J., Myers, B.A., Rothrock, B., 2006. UNIFORM: automatically generating consistent remote control user interfaces, in: Proceedings of the SIGCHI Conference on Human Factors in Computing Systems. pp. 611-620. doi:10.1145/1124772.1124865

Önüt, S., Tuzkaya, U.R., Doğaç, B., 2008. A particle swarm optimization algorithm for the multiple-level warehouse layout design problem. Comput. Ind. Eng. 54, 783-799. doi:10.1016/j.cie.2007.10.012

Oulasvirta, A., 2017a. Optimizing user interfaces for human performance, in: Lecture Notes in Computer Science (Including Subseries Lecture Notes in Artificial Intelligence and Lecture Notes in Bioinformatics). Springer, Cham, pp. 3-7. doi:10.1007/978-3-319-72038-8_1 
Oulasvirta, A., 2017b. User interface design with combinatorial optimization. Computer (Long. Beach. Calif). 50, 40-47. doi:10.1109/MC.2017.6

Oulasvirta, A., Kristensson, P.O., Bi, X., Howes, A. (Eds.), 2018. Computational Interaction. Oxford University Press. doi:10.1093/oso/9780198799603.001.0001

Pernice, K., Nielsen, J., Usability, E., Using, S., 2009. Eyetracking Methodology: How to Conduct and Evaluate Usability Studies Using Eyetracking. Notes 1-17.

Raeisi, S., Osqueizadeh, R., Maghsoudipour, M., Jafarpisheh, A.S., 2016. Ergonomic redesign of an industrial control panel. Int. J. Occup. Environ. Med.

Saifullah Hussin, M., Stützle, T., 2014. Tabu search vs. simulated annealing as a function of the size of quadratic assignment problem instances. Comput. Oper. Res. 43, 286-291. doi:10.1016/j.cor.2013.10.007

Sanders, M.S., McCormick, E.J., 1993. Human factors in engineering and design, New York: McGraw-Hill .

Srinivas, M., Patnaik, L.M., 1994. Genetic algorithms: a survey. Computer (Long. Beach. Calif). 27. doi:10.1109/2.294849

Stainer, M.J., Scott-Brown, K.C., Tatler, B.W., 2013. Looking for trouble: a description of oculomotor search strategies during live CCTV operation. Front. Hum. Neurosci. 7, 615. doi:10.3389/fnhum.2013.00615

Starke, S., Howes, A., Morar, N., Baber, C., 2015. Visual sampling in a road traffic management control room task, in: Sharples, S., Shorrock, S., Waterson, P. (Eds.), Proceedings of the International Conference on Ergonomics \& Human Factors 2015. Taylor \& Francis, Daventry, Northamptonshire, pp. 503-511.

Stewart, T., 1995. Ergonomics standards concerning human-system interaction. Appl. Ergon. 26, $271-274$. doi:10.1016/0003-6870(95)00031-7

Tam, K.Y., 1992. Genetic algorithms, function optimization, and facility layout design. Eur. J. Oper. Res. 63, 322-346. doi:10.1016/0377-2217(92)90034-7

Titz, J., Scholz, A., Sedlmeier, P., 2018. Comparing eye trackers by correlating their eye-metric data. Behav. Res. Methods 50, 1853-1863. doi:10.3758/s13428-017-0954-y

Todi, K., Jokinen, J., Luyten, K., Oulasvirta, A., 2018. Familiarisation: Restructuring Layouts with Visual Learning Models, in: Proceedings of the 2018 Conference on Human Information Interaction\&Retrieval IUI ’18. ACM Press, New York, New York, USA, pp. 547-558. doi:10.1145/3172944.3172949

Tompkins, J.A., White, J.A., Bozer, Y.A., Tanchoco, J.M.A., 2010. Facilities Planning, 4th ed. John Wiley \& Sons.

Tullis, T., Albert, B., 2013. Measuring the User Experience: Collecting, Analyzing, and Presenting Usability Metrics: Second Edition, Measuring the User Experience: Collecting, Analyzing, and Presenting Usability Metrics: Second Edition. doi:10.1016/C2011-0-00016-9

Wedel, M., 2017. Improving Ad Interfaces with Eye Tracking, in: The Wiley Handbook of Human Computer 
Interaction. John Wiley \& Sons, Ltd, Chichester, UK, pp. 889-907. doi:10.1002/9781118976005.ch41

Wickens C. D., M.J.S., 2008. Applied attention theory., Ergonomics. doi:10.1080/00140130802295564

Wickens, C.D., Carswell, C.M., 1995. The Proximity Compatibility Principle: Its Psychological Foundation and Relevance to Display Design. Hum. Factors J. Hum. Factors Ergon. Soc. 37, 473-494.

doi:10.1518/001872095779049408

Wickens, C.D., Helleberg, J., Xu, X., 2002. Pilot maneuver choice and workload in free flight. Hum. Factors J. Hum. Factors Ergon. Soc. 44, 171-88. doi:10.1518/0018720024497943

Wickens, C.D., Lee, J., Liu, Y.D., Gordon-Becker, S., 2003. An introduction to human factors engineering, 2nd editio. ed. Prentice-Hall, Inc., Upper Saddle River, NJ.

Wu, C., Liu, Y., 2009. Development and evaluation of an ergonomic software package for predicting multipletask human performance and mental workload in human-machine interface design and evaluation. Comput. Ind. Eng. 56, 323-333. doi:10.1016/j.cie.2008.06.013

Zhu, T., Balakrishnan, J., Cheng, C.H., 2017. Recent advances in dynamic facility layout research. INFOR Inf. Syst. Oper. Res. 1-29. doi:10.1080/03155986.2017.1363591 